\title{
p-ADIC HYPERBOLIC PLANES AND MODULAR FORMS
}

\author{
JOHN A. RHODES
}

\begin{abstract}
For $K$ a number field and $\mathrm{p}$ a finite prime of $K$, we define a p-adic hyperbolic plane and study its geometry under the action of $G L_{2}\left(K_{\mathrm{p}}\right)$. Seeking an operator with properties analogous to those of the non-Euclidean Laplacian of the classical hyperbolic plane, we investigate the fundamental invariant integral operator, the Hecke operator $T_{\mathrm{p}}$. Letting $S$ be a finite set of primes of a totally real $K$ (including all the infinite ones), a modular group $\Gamma(S)$ is defined. This group acts discontinuously on a product of classical and p-adic hyperbolic planes. $S$-modular forms and their associated Dirichlet series are studied.
\end{abstract}

\section{INTRODUCTION}

In [St], Stark initiated the study of $\mathbf{p}$-adic hyperbolic planes for application to modular forms. In this paper we continue the approach, developing Hecke operators which are then used to study Hilbert modular forms.

Of course there are already alternative approaches to Hecke theory for Hilbert modular forms. The oldest goes back to de Bruijn's work $[\mathrm{dB}]$ in defining a Hecke operator for any ideal that could be expressed as a principal ideal times the square of an ideal. However, this suffices only for those fields with odd class number $h$.

Herrmann remedied this situation in $[\mathrm{H}]$ by considering vectors of modular forms with $h$ components, each component modular with respect to a different group arising from its associated ideal class. His Hecke operators, defined for every integral ideal, acted on the entire vector, transforming each component as well as interchanging their order. His work is rather involved reading, though, primarily due to the use of ideal numbers as a bookkeeping device throughout.

The more modern introduction of adele groups into the theory of modular forms gave an alternative way of viewing Hecke operators that, at least in language if not in content, is far removed from Herrmann's approach (cf. [W]). While the adelic formulation is quite clean, some find it forbidding and the connections to the classical theory are not always obvious.

In presenting yet another approach we hope, as Stark says, to help "bridge the chasm separating the classical and modern points of view regarding modular forms and Dirichlet series." While it retains the use of hyperbolic planes

Received by the editors December 9, 1991.

1991 Mathematics Subject Classification. Primary 11F55; Secondary 11F41. 
throughout, the approach presented here has obvious connections to the adelic formulation.

\section{THE p-ADIC HYPERBoliC PLANE}

Let $K_{\mathrm{p}}$ be the completion of a number field, $K$, with respect to a prime ideal, p. $O_{\mathbf{p}}$ and $U_{\mathbf{p}}$ will denote the p-adic integers and units, respectively, in $K_{\mathbf{p}}$. The closure of $\mathbf{p}$ in $K_{\mathbf{p}}$ will also be denoted by $\mathbf{p}$. Choose any $\pi \in K_{\mathbf{p}}$ generating the ideal $\mathbf{p}$ and fix this choice for the remainder of this paper. We normalize the p-adic valuation so that $|\pi|_{\mathbf{p}}=1 / N_{Q}^{K}(\mathbf{p})=1 / p^{f}$, where $p$ is the rational prime over which $\mathbf{p}$ lies and $f$ is the residue degree of $\mathbf{p}$ over $p$. If $x \in K_{\mathbf{p}}^{\times}$, then by $\operatorname{ord}_{\mathbf{p}}(x)$ is meant the integer $n$ such that $x / \pi^{n} \in U_{\mathbf{p}}$. Finally, let $\left\{\alpha_{i}\right\}_{i=1}^{p^{f}}$ be a complete set of representatives of the residue class field $O_{\mathbf{p}} / \mathbf{p}$.

Our first goal is to define what is meant by the p-adic hyperbolic plane. Stark [St], proceeding in analogy with the complex upper half-plane, fixed some nonsquare unit, $\Delta$, of $K_{\mathbf{p}}$ and considered the set $\left\{x+\sqrt{\Delta} y \mid x, y \in K_{\mathbf{p}}, y \neq 0\right\}$. More generally, recall that every p-adic field has a unique unramified extension of any given degree (see [La] for instance). Let $L_{\mathbf{p}}$ denote the unramified extension of $K_{\mathrm{p}}$ of degree 2. Then its integers $O\left(L_{\mathbf{p}}\right)$ are an $O_{\mathbf{p}}$ module. Furthermore, some element $I$ of $O\left(L_{\mathbf{p}}\right)$ can be found such that $\{1, I\}$ is an $O_{\mathrm{p}}$ basis for $O\left(L_{\mathrm{p}}\right)$. Of course $I$ is not uniquely determined, so a choice must be made and fixed.

Definition. With $L_{\mathbf{p}}$ and $I$ as above, the (unramified) p-adic hyperbolic plane $H_{\mathbf{p}}$ is the set $L_{\mathbf{p}}-K_{\mathbf{p}}=\left\{x+I y \mid x, y \in K_{\mathbf{p}}, y \neq 0\right\}$ with the subspace topology inherited from $L_{\mathbf{p}}$.

If $z=x+I y$ is in $H_{\mathrm{p}}$, it will be useful to use $x(z)$ and $y(z)$ for the analogs of the real and imaginary parts of $z$.

Given a matrix $A=\left(\begin{array}{ll}a & b \\ c & d\end{array}\right) \in G L_{2}\left(K_{\mathbf{p}}\right)$, we associate to it the fractional linear transformation

$$
A \circ z=(a z+b) /(c z+d)
$$

for $z \in H_{\mathbf{p}}$. As in the classical case of the complex half-plane, a simple calculation shows that

$$
y(A \circ z)=(a d-b c) y(z) / \mathbf{N}_{K_{\mathrm{p}}}^{L_{\mathrm{p}}}(c z+d) .
$$

Thus if $y(z) \neq 0$, then $y(A \circ z) \neq 0$ for any $A \in G L_{2}\left(K_{\mathbf{p}}\right)$. Since another calculation shows that multiplication of group elements corresponds to composition of transformations, the above definition of $A \circ z$ defines a group action of $G L_{2}\left(K_{\mathbf{p}}\right)$ on $H_{\mathbf{p}}$. Clearly this action is transitive, as $\left(\begin{array}{ll}y & x \\ 0 & 1\end{array}\right) \circ I=x+I y$. It is also useful to know what elements of the group fix $I$.

Lemma 1. The isotropy group of $I$ in $G L_{2}\left(K_{\mathrm{p}}\right)$ is

$$
\left\{\left(\begin{array}{cc}
d+c \operatorname{Tr}_{K_{\mathrm{p}}}^{L_{\mathrm{p}}}(I) & -c \mathbf{N}_{K_{\mathrm{p}}}^{L_{\mathrm{p}}}(I) \\
c & d
\end{array}\right) \mid d, c \in K_{\mathrm{p}} \text {, not both zero }\right\} .
$$

Proof. $A \circ I=\left(\begin{array}{ll}a & b \\ c & d\end{array}\right) \circ I=I$ means that $a I+b=I(c I+d)$. Using $I^{2}-$ $\operatorname{Tr}_{K_{\mathrm{p}}}^{L_{\mathrm{p}}}(I) I+\mathbf{N}_{K_{\mathrm{p}}}^{L_{\mathrm{p}}}(I)=0$, we find that $a I+b=\left(d+c \operatorname{Tr}_{K_{\mathrm{p}}}^{L_{\mathrm{p}}}(I)\right) I-c \mathbf{N}_{K_{\mathrm{p}}}^{L_{\mathrm{p}}}(I)$. Since $\{1, I\}$ is a basis for $L_{\mathbf{p}}$ over $K_{\mathrm{p}}$, we can now equate the " $x$ " and " $y$ " parts of this last equation to show $A$ has the form given above. Such a matrix has determinant $d^{2}+c d \operatorname{Tr}_{K_{\mathrm{p}}}^{L_{\mathrm{p}}}(I)+c^{2} \mathbf{N}_{K_{\mathrm{p}}}^{L_{\mathrm{p}}}(I)$. Suppose this were zero. Then factoring 
this quadratic in $L_{\mathbf{p}}$ shows $(d+c I)(d+c \bar{I})=0$, where the bar denotes taking conjugates in $L_{\mathbf{p}}$ over $K_{\mathbf{p}}$. This is of course impossible, unless both $c$ and $d$ are zero.

The isotropy group oi $I$ is actually abelian. In fact, there is an isomorphism of the isotropy group with $L_{\mathbf{p}}^{\times}$given by

$$
\left(\begin{array}{cc}
d+c \operatorname{Tr}_{K_{\mathbf{p}}}^{L_{\mathrm{p}}}(I) & -c \mathbf{N}_{K_{\mathrm{p}}}^{L_{\mathrm{p}}}(I) \\
c & d
\end{array}\right) \mapsto d+I c .
$$

Moreover this is a homeomorphism and the determinant of the matrix above is just $\mathbf{N}_{K_{\mathbf{p}}}^{L_{\mathbf{p}}}(d+I c)$. This is in complete analogy to the complex upper half-plane case where the isotropy group of $i$ in $G L_{2}(\mathbf{R})$ is composed of elements of the form $\left(\begin{array}{cc}x & -y \\ y & x\end{array}\right)$, and this matrix is just the image of $x+i y$ under the usual embedding of $\mathbf{C}^{\times}$in $G L_{2}(\mathbf{R})$. $H_{\mathbf{p}}$, of course, could be viewed as $G$ modulo the isotropy group of $I$.

$H_{\mathrm{p}}$ has the topology it inherited from $L_{\mathrm{p}}$, which arises from extending the p-adic valuation of $K_{\mathbf{p}}$. A basis for the topology of $H_{\mathbf{p}}$ can be given, though, which behaves nicely under the action of $G L_{2}\left(K_{\mathbf{p}}\right)$.

First, recall that in $K_{\mathrm{p}}$ we have the filtrations

$$
\begin{aligned}
& O_{\mathbf{p}}=\mathbf{p}^{0} \supset \mathbf{p}^{1} \supset \mathbf{p}^{2} \supset \cdots \supset \mathbf{p}^{n} \supset \cdots, \\
& U_{\mathbf{p}}=U_{0} \supset U_{1} \supset U_{2} \supset \cdots \supset U_{n} \supset \cdots,
\end{aligned}
$$

where $U_{n}=\left\{\varepsilon \in U_{\mathbf{p}} \mid \varepsilon \equiv 1\left(\bmod \mathbf{p}^{n}\right)\right\}$ for $n \geq 1$. The first is just a fundamental system of neighborhoods of 0 in $K_{\mathbf{p}}$, while the second (from which we omit a subscript $\mathbf{p}$ ) is a fundamental system of neighborhoods of 1 in $K_{\mathbf{p}}^{\times}$.

Definition. If $z=x+I y \in H_{\mathbf{p}}$, let

$$
M_{z}^{n}=\left\{u+I v \in H_{\mathbf{p}} \mid(u-x) / y \in \mathbf{p}^{n} \text { and } v / y \in U_{n}\right\} .
$$

Clearly $M_{z}^{0} \supset M_{z}^{1} \supset M_{z}^{2} \supset \cdots$. Note that $M_{z}^{n}$ is unchanged if $y$ is replaced by $\varepsilon y$ where $\varepsilon \in U_{n}$ or if $x$ is replaced by $x+y \alpha$ where $\alpha \in \mathbf{p}^{n}$. In fact $\xi \in M_{z}^{n}$ if and only if $M_{z}^{n}=M_{\xi}^{n}$. Thus any $M_{z}^{n}$ and $M_{\xi}^{n}$ are either identical or disjoint.

Lemma 2. The $M_{z}^{n}$ form a basis for the topology of $H_{\mathbf{p}}$.

Proof. Since $L_{\mathbf{p}}$ is unramified over $K_{\mathbf{p}}$ and $\{1, I\}$ is an $O_{\mathbf{p}}$ basis for $O\left(L_{\mathbf{p}}\right)$, then the valuation in $L_{\mathrm{p}}$ of $x+I y$, with $x, y \in K_{\mathrm{p}}$, is just $|x+I y|_{\mathrm{p}}=$ $\max \left\{|x|_{\mathbf{p}},|y|_{\mathbf{p}}\right\}$. Thus two points in $H_{\mathbf{p}}$ will be close if and only if both their $x$ parts and $y$. parts are p-adically close. But if $z=x+I y$ and $\xi=u+I v$, the statement that $\xi \in M_{z}^{n}$ is exactly that $x-u \in y \mathbf{p}^{n}$ and $y-v \in y \mathbf{p}^{n}$.

Although it can be shown by direct calculation that if $A \in G L_{2}\left(K_{\mathbf{p}}\right)$ and $z \in H_{\mathrm{p}}$ then $A \circ M_{z}^{n}=M_{A \circ z}^{n}$, that proof is rather unenlightening. Instead we give a proof following several intermediate lemmas, which will be of use in their own right as well.

Lemma 3. Let $A \in G L_{2}\left(K_{\mathbf{p}}\right), \operatorname{det}(A) \in U_{\mathbf{p}}$. Then $A \circ I \in M_{I}^{n}$ if and only if $A \in G L\left(O_{\mathbf{p}}\right)$ and for some $c, d \in K_{\mathbf{p}}$

$$
A \equiv\left(\begin{array}{cc}
d+c \operatorname{Tr}_{K_{\mathrm{p}}}^{L_{\mathrm{p}}}(I) & -c \mathbf{N}_{K_{\mathrm{p}}}^{L_{\mathrm{p}}}(I) \\
c & d
\end{array}\right) \quad\left(\bmod \mathbf{p}^{n}\right) .
$$


Proof. First assume $A=\left(\begin{array}{ll}a & b \\ c & d\end{array}\right) \in G L_{2}\left(O_{\mathbf{p}}\right)$ satisfies the congruence above. Then calculating

$$
A \circ I=\frac{b d+b c \operatorname{Tr}_{K_{\mathrm{p}}}^{L_{\mathrm{p}}}(I)+a c \mathbf{N}_{K_{\mathrm{p}}}^{L_{\mathrm{p}}}(I)+I(a d-b c)}{c^{2} \mathbf{N}_{K_{\mathrm{p}}}^{L_{\mathrm{p}}}(I)+c d \operatorname{Tr}_{K_{\mathrm{p}}}^{L_{\mathrm{p}}}(I)+d^{2}},
$$

we see that the denominator here is congruent to $\operatorname{det}(A)\left(\bmod \mathbf{p}^{n}\right)$, and hence $y(A \circ I) \in U_{n}$. Also one sees that $b d+b c \operatorname{Tr}_{K_{\mathrm{p}}}^{L_{\mathrm{p}}}(I)+a c \mathbf{N}_{K_{\mathrm{p}}}^{L_{\mathrm{p}}}(I) \equiv 0\left(\bmod \mathbf{p}^{n}\right)$, and so $x(A \circ I) \in \mathbf{p}^{n}$. Therefore $A \circ I \in M_{I}^{n}$.

Conversely, if $A \circ I \in M_{I}^{n}, \operatorname{det}(A) \in U_{\mathrm{p}}$, let $x+I y=A \circ I$. Then $\left(\begin{array}{cc}1 & -x \\ 0 & y\end{array}\right) A$ is in the isotropy group of $I$. So by Lemma 1

$$
\left(\begin{array}{cc}
1 & -x \\
0 & y
\end{array}\right) A=\left(\begin{array}{cc}
d+c \operatorname{Tr}_{K_{\mathrm{p}}}^{L_{\mathrm{p}}}(I) & -c \mathbf{N}_{K_{\mathrm{p}}}^{L_{\mathrm{p}}}(I) \\
c & d
\end{array}\right)
$$

for some $c, d$ not both zero.

Since $y \in U_{n}$, the determinant of the left-hand side is a unit. But the determinant of the right-hand side is just $\mathbf{N}_{K_{\mathrm{p}}}^{L_{\mathrm{p}}}(d+I c)$. Therefore $d$ and $c$ are actually integral and the right-hand side is in $G L_{2}\left(O_{p}\right)$. Thus

$$
A=\left(\begin{array}{cc}
1 & -x \\
0 & y
\end{array}\right)^{-1}\left(\begin{array}{cc}
d+c \operatorname{Tr}_{K_{\mathrm{p}}}^{L_{\mathrm{p}}}(I) & -c \mathbf{N}_{K_{\mathrm{p}}}^{L_{\mathrm{p}}}(I) \\
c & d
\end{array}\right) \in G L_{2}\left(O_{\mathbf{p}}\right)
$$

and since

$$
\left(\begin{array}{cc}
1 & -x \\
0 & y
\end{array}\right) \equiv\left(\begin{array}{ll}
1 & 0 \\
0 & 1
\end{array}\right) \quad\left(\bmod \mathbf{p}^{n}\right)
$$

$A$ satisfies the stated congruence.

Corollary 4. Let $A \in G L_{2}\left(K_{\mathrm{p}}\right)$. Then $A \circ M_{I}^{n}=M_{I}^{n}$ if and only if

$$
A=\left(\begin{array}{cc}
\pi^{m} & 0 \\
0 & \pi^{m}
\end{array}\right) B
$$

where

$$
B \in G L_{2}\left(O_{\mathrm{p}}\right), \quad B \equiv\left(\begin{array}{cc}
d+c \operatorname{Tr}_{K_{\mathrm{p}}}^{L_{\mathrm{p}}}(I) & -c \mathbf{N}_{K_{\mathrm{p}}}^{L_{\mathrm{p}}}(I) \\
c & d
\end{array}\right) \quad\left(\bmod \mathbf{p}^{n}\right) .
$$

Proof. First assume $\operatorname{det}(A) \in U_{\mathbf{p}}$. Then $A \circ M_{I}^{n}=M_{I}^{n}$ implies that in particular $A \circ I \in M_{I}^{n}$, so by Lemma 3 the congruence is established. Conversely, if $A$ satisfies the congruence and $z=x+I y \in M_{I}^{n}$, then $A \circ z=A\left(\begin{array}{ll}y & x \\ 0 & 1\end{array}\right) \circ I$. But since $\left(\begin{array}{ll}y & x \\ 0 & 1\end{array}\right)$ is congruent to the identity $\bmod \mathbf{p}^{n}$, we see $A\left(\begin{array}{ll}y & x \\ 0 & 1\end{array}\right)$ satisfies the congruences of Lemma 3 , and hence $A\left(\begin{array}{ll}y & x \\ 0 & 1\end{array}\right) \circ I \in M_{I}^{n}$, so $A \circ M_{I}^{n} \subset M_{I}^{n}$. The reverse inclusion comes from considering $A^{-1}$.

If $\operatorname{ord}_{\mathbf{p}}(\operatorname{det}(A))$ is even, rescaling by $\left(\begin{array}{cc}\pi^{m} & 0 \\ 0 & \pi^{m}\end{array}\right)$ for appropriate $m$ reduces this case to the first.

Finally, the case $\operatorname{ord}_{\mathbf{p}}(\operatorname{det}(A))$ odd does not occur. For if $A=\left(\begin{array}{ll}a & b \\ c & d\end{array}\right)$ and $A \circ I=x+I y$, then $y=\operatorname{det}(A) / \mathbf{N}_{K_{\mathrm{p}}}^{L_{\mathrm{p}}}(d+I c) . \operatorname{But} \operatorname{ord}_{\mathrm{p}}\left(\mathbf{N}_{K_{\mathrm{p}}}^{L_{\mathrm{p}}}(d+I c)\right)$ is even since $L_{\mathrm{p}}$ is unramified over $K_{\mathrm{p}}$. Thus $\operatorname{ord}_{\mathrm{p}}(\operatorname{det}(A))$ is odd implies $\operatorname{ord}_{\mathrm{p}}(y)$ is odd, so that $y \notin U_{n}$ so $A \circ I \notin M_{I}^{n}$.

The reason that we have taken $L_{\mathrm{p}}$ to be an unramified extension of $K_{\mathrm{p}}$ is just so that this corollary would hold. 
Proposition 5. If $A \in G L_{2}\left(K_{\mathrm{p}}\right)$, then $A \circ M_{z}^{n}=M_{A \circ z}^{n}$. That is, the $M_{z}^{n}$ form a $G L_{2}\left(K_{\mathrm{p}}\right)$-invariant basis for the topology of $H_{\mathrm{p}}$.

Proof. If $z=x+I y$, it follows easily from the definition of $M_{I}^{n}$ that $\left(\begin{array}{ll}y & x \\ 0 & 1\end{array}\right) \circ$ $M_{I}^{n}=M_{z}^{n}$. Therefore, once it is shown that $B \circ M_{I}^{n}=M_{B \circ I}^{n}$, the proposition will follow since then

$$
A \circ M_{z}^{n}=A\left(\begin{array}{ll}
y & x \\
0 & 1
\end{array}\right) \circ M_{I}^{n}=M_{A\left(\begin{array}{ll}
y & x \\
0 & 1
\end{array}\right) \circ I}^{n}=M_{A \circ z}^{n} .
$$

Now $M_{I}^{n}=C \circ M_{I}^{n}$ for any $C$ satisfying the requirements of Corollary 4, so $B \circ M_{I}^{n}=B C \circ M_{I}^{n}$. But if $B=\left(\begin{array}{l}a b \\ c d\end{array}\right)$, then such a $C$ can be found so that $B C=\left(\begin{array}{ll}y & x \\ 0 & 1\end{array}\right)$. This is done as follows: Let $e=-d \pi^{m}$, and $g=c \pi^{m}$, where $m$ is minimal such that $e$ and $g$ are integral. Solving the congruences $e \equiv h+g \operatorname{Tr}_{K_{\mathrm{p}}}^{L_{\mathrm{p}}}(I)\left(\bmod \mathbf{p}^{n}\right)$ and $f \equiv-\mathbf{N}_{K_{\mathrm{p}}}^{L_{\mathrm{p}}}(I) g\left(\bmod \mathbf{p}^{n}\right)$ for $h$ and $f$, we can construct a matrix $\left(\begin{array}{l}e f \\ g h\end{array}\right)$ in $G L_{2}\left(O_{\mathbf{p}}\right)$ satisfying the congruences of the preceding corollary. Furthermore

$$
\left(\begin{array}{ll}
a & b \\
c & d
\end{array}\right)\left(\begin{array}{ll}
e & f \\
g & h
\end{array}\right)=\left(\begin{array}{ll}
r & s \\
0 & t
\end{array}\right) \text { for some } r, s, \text { and } t .
$$

Multiplying $\left(\begin{array}{ll}e & f \\ g & h\end{array}\right)$ by $\left(\begin{array}{cc}1 / t & 0 \\ 0 & 1 / t\end{array}\right)$ gives a matrix $C$ with all the desired properties. Therefore

$$
B \circ M_{I}^{n}=B C \circ M_{I}^{n}=\left(\begin{array}{cc}
y & x \\
0 & 1
\end{array}\right) \circ M_{I}^{n}=M_{z}^{n}
$$

for some $z$. Finally, since $B \circ I \in M_{z}^{n}$, and $M_{z}^{n}$ is just as well indexed by any of its elements as by $z$, we have $B \circ M_{I}^{n}=M_{B \circ I}^{n}$.

Actually, for each $n \geq 0$ the proofs above give a decomposition theorem for the group $G L_{2}\left(K_{\mathrm{p}}\right)$ (or even $G L_{2}(K)$ ). Namely, any matrix $B$ in the groups can be written $\left(\begin{array}{ll}y & x \\ 0 & 1\end{array}\right) A C$ where $C \in G L_{2}\left(O_{\mathbf{p}}\right)$ is congruent $\bmod \mathbf{p}^{n}$ to an element of the isotropy group of $I$ and $A$ is in the center of the group.

Since the compact sets $M_{z}^{n}$ for fixed $n$ are permuted by the action of $G L_{2}\left(K_{\mathrm{p}}\right)$, there is a $G L_{2}(K)$-invariant measure on $H_{\mathrm{p}}$, denoted by $\mu$, satisfying

$$
\begin{gathered}
\mu\left(M_{z}^{0}\right)=1, \quad \mu\left(M_{z}^{1}\right)=1 / \mathbf{N}_{Q}^{K}(\mathbf{p})\left(\mathbf{N}_{Q}^{K}(\mathbf{p})-1\right), \\
\mu\left(M_{z}^{n+1}\right)=\mu\left(M_{z}^{n}\right) / \mathbf{N}_{Q}^{K}(\mathbf{p})^{2} \quad \text { for } n \geq 1 .
\end{gathered}
$$

When modular forms are defined below in such a way as to extend Hilbert modular forms, they will necessarily be constant on sets of the form $M_{z}^{n}$, for some fixed $n$. The measure defined here, then, will be the appropriate one with which to integrate them.

Definition. A function defined on $H_{\mathrm{p}}$ is called locally constant of order $n$ if it is constant on all sets of the form $M_{z}^{n}$.

When defining modular forms on products of the complex upper half-plane, one requires that they be eigenfunctions of certain differential operators. The 
operators considered are the "simplest" ones that commute with the slash operator. It is only by requiring that modular forms be eigenfunctions of these operators that the full shape of their Fourier series can be determined, and hence the gamma factors of the associated $L$-series. An analogous course will be followed for the p-adic plane.

Definition. If $f: H_{\mathbf{p}} \rightarrow C$, then the slash operator of weight $k$ is defined by

$$
\left.f\right|_{\mathbf{p}, k} A(z)=\left.f\right|_{k} A(z)=f(A \circ z)\left(|c z+d|_{\mathbf{p}}\right)^{-k}\left(|\operatorname{det}(A)|_{\mathbf{p}}\right)^{k / 2}
$$

where $A=\left(\begin{array}{ll}a & b \\ c & d\end{array}\right) \in G L_{2}\left(K_{\mathbf{p}}\right)$.

One checks that $\left.\left.f\right|_{k} A\right|_{k} B=\left.f\right|_{k}(A B)$ and that if $f$ is locally constant of order $n$ then so is $\left.f\right|_{k} A$.

Note that

$$
|y|_{\mathbf{p}}^{k / 2}\left(\left.f\right|_{k} A\right)=\left.\left(|y|_{\mathbf{p}}^{k / 2} f\right)\right|_{0} A
$$

so we would lose no generality by only considering the weight 0 slash operator. While the concept of weight is essentially fictional in this context, it will be convenient later on.

We are now interested in operators $T$ acting on functions defined on $H_{\mathrm{p}}$ such that

$$
T\left(\left.f\right|_{k} A\right)=\left.(T f)\right|_{k} A \text { for all } A \in G L_{2}\left(K_{\mathbf{p}}\right) .
$$

In the complex half-plane case, the non-Euclidean weight $k$ "Laplacian" is such an operator. However, since there is no point in differentiating locally constant functions, this does not help us in the p-adic case. On the other hand, in order to study eigenfunctions of differential operators, it is standard to look at related integral operators and integral operators can be investigated in the p-adic case.

Definition. If $g(\xi, z): H_{\mathrm{p}} \times H_{\mathrm{p}} \rightarrow \mathrm{C}$ is locally constant of order $n$ in both variables, then let the operator $T_{g}$ be defined by

$$
\left(T_{g} f\right)(z)=\int_{H_{\mathbf{p}}} f(\xi) g(\xi, z) d \mu(\xi)
$$

whenever this makes sense. (Here $d \mu$ is the invariant measure defined above.)

Definition. $h(\xi, z): H_{\mathbf{p}} \times H_{\mathbf{p}} \rightarrow \mathbf{C}$ is called a point-pair invariant if

$$
h(A \circ \xi, A \circ z)=h(\xi, z) \text { for all } A \in G L_{2}\left(K_{\mathrm{p}}\right) .
$$

Lemma 6. Let $z=x+I y$ and $\xi=u+I v$. Then $T_{g}$ commutes with the weight $k$ slash operator for all compactly supported locally constant functions of order $n$ if and only if $g_{1}(\xi, z)=|y / v|_{\mathbf{p}}^{k / 2} g(\xi, z)$ is a point-pair invariant.

Proof. Let $A=\left(\begin{array}{ll}a & b \\ c & d\end{array}\right) \in G L_{2}\left(K_{\mathbf{p}}\right)$. Then $\left.\left(T_{g} f\right)\right|_{k} A=T_{g}\left(\left.f\right|_{k} A\right)$ means that

$$
\begin{aligned}
\mid c z+ & \left.\left.d\right|_{\mathbf{p}} ^{-k} \operatorname{det}(A)\right|_{\mathbf{p}} ^{k / 2} \int_{H_{\mathbf{p}}} f(\xi) g(\xi, A \circ z) d \mu(\xi) \\
& =\int_{H_{\mathbf{p}}}|c \xi+d|_{\mathbf{p}}^{-k}|\operatorname{det}(A)|_{\mathbf{p}}^{k / 2} f(A \circ \xi) g(\xi, z) d \mu(\xi)
\end{aligned}
$$


or equivalently, by using the invariance of $\mu$, that

$$
\begin{aligned}
\mid c z+ & \left.d\right|_{\mathbf{p}} ^{-k} \int_{H_{\mathbf{p}}} f(A \circ \xi) g(A \circ \xi, A \circ z) d \mu(\xi) \\
& =\int_{H_{\mathbf{p}}}|c \xi+d|_{\mathbf{p}}^{-k} f(A \circ \xi) g(\xi, z) d \mu(\xi) .
\end{aligned}
$$

This holds for all $f$ if and only if

$$
|c z+d|_{\mathbf{p}}^{-k} g(A \circ \xi, A \circ z)=|c \xi+d|_{\mathbf{p}}^{-k} g(\xi, z) \text {. }
$$

That is, $|y / v|_{\mathbf{p}}^{k / 2} g(\xi, z)$ is a point-pair invariant.

Now it is clear that we should look for locally-constant of order $n$ pointpair invariants. If $g_{1}(\xi, z)$ is such a function, then it gives rise to a function $g_{0}(\xi)=g_{1}(\xi, I)$. With certain conditions imposed on $g_{0}$ the process can be reversed.

Lemma 7. Given $g_{0}: H_{\mathbf{p}} \rightarrow \mathrm{C}$ locally constant of order $n$, define $g_{1}(\xi, z)=$ $g_{0}(\xi)$ for $z \in M_{I}^{n}$. Then $g_{1}$ extends to a locally constant of order $n$ point-pair invariant if and only if $g_{0}(\xi)=g_{0}(A \circ \xi)$ for all $A$ such that $A \circ M_{I}^{n}=M_{I}^{n}$.

Proof. First suppose $g_{1}$ extends to a locally-constant point-pair invariant and $A \circ M_{I}^{n}=M_{I}^{n}$. Then $A^{-1} \circ M_{I}^{n}=M_{I}^{n}$ so $g_{1}(\xi, I)=g_{1}\left(\xi, A^{-1} \circ I\right)=g_{1}(A \circ \xi, I)$, so $g_{0}(\xi)=g_{0}(A \circ \xi)$.

Conversely, suppose $g_{0}(\xi)=g_{0}(A \circ \xi)$ for all $A$ fixing $M_{I}^{n}$ as a set and define $g_{1}(\xi, z)=g_{1}(A \circ \xi, A \circ z)=g_{0}(A \circ \xi)$ where $A$ is chosen so that $A \circ z \in M_{I}^{n}$. If $g_{1}$ is well defined, then by the transitivity of the action of $G L_{2}\left(K_{\mathrm{p}}\right)$ on $H_{\mathrm{p}}, g_{1}$ is defined on all of $H_{\mathrm{p}} \times H_{\mathrm{p}}$. Further, if $A \circ z \in M_{I}^{n}$, then $g_{1}(B \circ \xi, B \circ z)=g_{1}\left(A B^{-1}(B \circ \xi), A B^{-1}(B \circ z)\right)=g_{0}(A \circ \xi)$ by definition. Thus $g_{1}(B \circ \xi, B \circ z)=g_{1}(\xi, z)$ and $g_{1}$ is a point-pair invariant. It only remains to check consistency of the definition: for fixed $z, A$ and $B$ such that $A \circ z, B \circ z \in M_{I}^{n}$, we must show $g_{0}(A \circ \xi)=g_{0}(B \circ \xi)$. But $B A^{-1} \circ M_{I}^{n}=M_{I}^{n}$ so this follows at once from the hypothesis.

If we are interested in having our operators $T_{g}$, where $g_{1}(\xi, z)=$ $|y / v|_{\mathbf{p}}^{k / 2} g(\xi, z)$ is a point-pair invariant and $g_{0}(\xi)=g_{1}(\xi, I)$, act on all locally constant functions on $H_{\mathrm{p}}$, then it is necessary that the integral in the definition of $T_{g}$ reduce to a finite sum ultimately. That is, $g_{0}$ must have compact support. The obvious way to construct a compactly supported function $g_{0}$ such that $g_{0}(\xi)=g_{0}(A \circ \xi)$ for all $A$ in some group $G$ is through characteristic functions. More specifically, if a set $M$ in $H_{\mathrm{p}}$ is invariant under all matrices $A \in G$, then taking $g_{0}$ to be the characteristic function of $M$ gives a function of the type desired.

Lemma 8. Let $z=x+I y \in H_{\mathrm{p}}, G^{n}=\left\{A \mid A \circ M_{I}^{n}=M_{I}^{n}\right\}$ for some fixed $n$. If $G^{n}\left(\begin{array}{ll}y & x \\ 0 & 1\end{array}\right) G^{n}=\bigcup_{i} A_{i} G^{n}$ is a double coset decomposition, then $G^{n} \circ M_{z}^{n}=$ $\bigcup_{i} A_{i} \circ M_{I}^{n}$.

Proof. $\bigcup_{i} A_{i} \circ M_{I}^{n}=\bigcup_{i} A_{i} G^{n} \circ I$ since $M_{I}^{n}=G^{n} \circ I$. So

$$
\begin{aligned}
\bigcup_{i} A_{i} \circ M_{I}^{n} & =G^{n}\left(\begin{array}{cc}
y & x \\
0 & 1
\end{array}\right) G^{n} \circ I \\
& =G^{n}\left(\begin{array}{cc}
y & x \\
0 & 1
\end{array}\right) \circ M_{I}^{n}=G^{n} \circ M_{z}^{n} .
\end{aligned}
$$


Now given any point $z \in H_{\mathbf{p}}$, one can let $h_{0}$ be the characteristic function of $G^{n} \circ M_{z}^{n}$. Then via Lemmas 6 and 7 there is an associated integral operator $T_{h}$ that commutes with the weight $k$ slash operator. Of course the operators arising from two distinct $z$ will not necessarily be distinct. Already it is obvious that any integral operator $T_{g}$ associated to a compactly supported $g_{0}$ via the above lemmas will be a linear combination of the operators described here, since $g_{0}$ itself must be a linear combination of characteristic functions of sets of the form $G^{n} \circ M_{z}^{n}$. Since we are looking for an analog of the Laplacian for functions on $H_{\mathrm{p}}$, we would like to find a single fundamental integral operator in terms of which all integral operators commuting with the weight $k$ slash operator can be expressed. In general, such an operator does not exist. However if we specialize to the case which will ultimately be most valuable anyway, namely the locally constant functions of order 0 , there is such an operator.

Therefore, for the remainder of this section all functions will be locally constant of order 0 , i.e. constant on sets of the form $M_{z}=M_{z}^{0}$. Recalling from Corollary 4 that $A \circ M_{I}=M_{I}$ if and only if $A=\left(\begin{array}{cc}\pi^{n} & 0 \\ 0 & \pi^{n}\end{array}\right) B$ where $B \in G L_{2}\left(O_{\mathrm{p}}\right)$, we let $G=G L_{2}\left(O_{\mathrm{p}}\right)$. Then $M_{I}=G \circ I$. The p-adic Laplacian is now easily defined.

Definition. Let $l_{0}$ be the characteristic function of the set

$$
G \circ M_{I \pi}=\bigcup_{i=1}^{p^{f}}\left(\begin{array}{cc}
\pi & \alpha_{i} \\
0 & 1
\end{array}\right) \circ M_{I} \cup\left(\begin{array}{ll}
1 & 0 \\
0 & \pi
\end{array}\right) \circ M_{I}
$$

Then if $T_{l}$ is the integral operator associated to $l_{0}$, let $T_{\mathbf{p}, k}=T_{\mathbf{p}}=|\pi|^{1-k / 2} T_{l}$. $T_{\mathbf{p}}$ will be called the weight $k$ Hecke operator for the plane $H_{\mathbf{p}}$.

Of course one must check that

$$
G\left(\begin{array}{cc}
\pi & 0 \\
0 & 1
\end{array}\right) G=\bigcup_{i=1}^{p^{f}}\left(\begin{array}{cc}
\pi & \alpha_{i} \\
0 & 1
\end{array}\right) G \cup\left(\begin{array}{ll}
1 & 0 \\
0 & \pi
\end{array}\right) G
$$

so that, by Lemma $8, G \circ M_{I \pi}$ really is as stated in the definition above. This is standard, so we omit the tedious verification. We now give a more explicit description of the way $T_{\mathbf{p}}$ acts on functions.

Lemma 9. If $z=x+I y$, then

$$
T_{\mathbf{p}} f(z)=|\pi|_{\mathbf{p}} \sum_{i=1}^{p^{f}} f\left(x+\alpha_{i} y+I \pi y\right)+|\pi|_{\mathbf{p}}^{1-k} f\left(x+I y \pi^{-1}\right) .
$$

Proof. Let $A=\left(\begin{array}{cc}1 & -x \\ 0 & y\end{array}\right) \in G L_{2}\left(K_{\mathrm{p}}\right)$ so $A \circ z=I$. Then $l_{1}(\xi, z)=l_{1}(A \circ \xi, I)=$ $l_{0}(A \circ \xi)$. Therefore

$$
l_{1}(\xi, z)= \begin{cases}1 & \text { if } \xi \in A^{-1} G \circ M_{I \pi} \\ 0 & \text { otherwise }\end{cases}
$$

Letting $\xi=u+I v$, then

$$
T_{\mathbf{p}} f(z)=|\pi|_{\mathbf{p}}^{1-k / 2} \int_{\xi \in A^{-1} G \circ M_{I \pi}} f(\xi)\left|\frac{v}{y}\right|_{\mathbf{p}}^{k / 2} d \mu(\xi) .
$$


Since

$$
A^{-1} G \circ M_{I \pi}=\bigcup_{i=1}^{p^{f}}\left(\begin{array}{cc}
y \pi & x+\alpha_{i} y \\
0 & 1
\end{array}\right) \circ M_{I} \cup\left(\begin{array}{cc}
y \pi^{-1} & x \\
0 & 1
\end{array}\right) \circ M_{I},
$$

then using that $f$ is locally constant

$$
T_{\mathbf{p}} f(z)=|\pi|_{\mathbf{p}}^{1-k / 2}\left[\sum_{i=1}^{p^{f}} f\left(x+\alpha_{i} y+I y \pi\right)|\pi|_{\mathbf{p}}^{k / 2}+f\left(x+I y \pi^{-1}\right)|\pi|_{\mathbf{p}}^{-k / 2}\right] .
$$

The key result concerning $T_{\mathbf{p}}$ is

Theorem 10. Any integral operator $T_{g}$ acting on functions on $H_{\mathrm{p}}$ which commutes with the weight $k$ slash operator and which is associated (via Lemmas 6 and 7) to a compactly supported $g_{0}$ can be expressed as a polynomial in $T_{\mathbf{p}}$ with complex coefficients.

In the course of proving this the following explicit double coset decomposition will be needed.

Lemma 11. If $n$ is a nonnegative integer and $\Delta^{n}$ denotes the set of $2 \times 2$ matrices with entries in $O_{\mathbf{p}}$ and determinant in $\mathbf{p}^{n}-\mathbf{p}^{n+1}$, then

$$
G \Delta^{n} G=\bigcup\left(\begin{array}{cc}
\pi^{j} & \beta \\
0 & \pi^{n-j}
\end{array}\right) G
$$

where the union is disjoint and taken over $j=0,1,2, \ldots, n$ and $\beta$ running through a complete set of representatives of $O_{\mathbf{p}} \bmod \mathbf{p}^{j}$.

Proof. The proof is exactly as the analogous fact for double cosets of $S L_{2}(\mathbf{Z})$ used in standard treatments (e.g., [Sh]) of the Hecke operators, and is therefore omitted. We only note that since we are taking left coset representatives instead of the usual right coset representatives, we have $\beta$ mod $\mathbf{p}^{j}$ rather than $\bmod \mathbf{p}^{n-j}$.

Proof of Theorem 10. The proof will proceed by induction, but first the inductive parameter must be defined. Given a nonzero operator $T_{g}$ as in the statement of the theorem, let $m(g)$ be the largest integer $m$ such that $g_{0}\left(I \pi^{m}\right) \neq 0$. It must be shown that $m(g)$ exists since it is conceivable that $g_{0}\left(I \pi^{m}\right)=0$ for all $m$ even though $T_{g}$ is not identically zero.

We claim that in fact $m(g) \geq 0$ and $m(g)=0$ if and only if $g_{0}$ vanishes outside of $M_{I}$. We will repeatedly use that $g_{0}(z) \neq 0$ implies that $g_{0}(\xi) \neq 0$ for any

$$
\xi \in G \circ M_{z}=G\left(\begin{array}{cc}
y & x \\
0 & 1
\end{array}\right) \circ M_{I}=G\left(\begin{array}{cc}
y & x \\
0 & 1
\end{array}\right)\left(\begin{array}{cc}
\pi^{n} & 0 \\
0 & \pi^{n}
\end{array}\right) G \circ I .
$$

Let $z$ be any point such that $g_{0}(z) \neq 0$. Then replacing $z$ by $\left(\begin{array}{cc}0 & 1 \\ -1 & 0\end{array}\right) z$ if necessary, we may assume $\operatorname{ord}_{p}(y) \geq 0$. Also, by replacing $z$ by $\left(\begin{array}{ll}1 & 1 \\ 0 & 1\end{array}\right) z$ if necessary, we may assume $\operatorname{ord}_{\mathbf{p}}(x) \leq 0$. If $\operatorname{ord}_{\mathbf{p}}(y)=\operatorname{ord}_{\mathbf{p}}(x)=0$, then $x+I y \in M_{I}$ so $m(g) \geq 0$. Otherwise, let $n=-\operatorname{ord}_{\mathrm{p}}(x)$ so that

$$
\left(\begin{array}{cc}
y & x \\
0 & 1
\end{array}\right)\left(\begin{array}{cc}
\pi^{n} & 0 \\
0 & \pi^{n}
\end{array}\right)=\left(\begin{array}{cc}
\alpha & \beta \\
0 & \gamma
\end{array}\right)
$$


where $\alpha, \gamma \in O_{\mathbf{p}}$ and $\beta \in U_{\mathbf{p}}$. Then

$$
G\left(\begin{array}{cc}
\alpha & \beta \\
0 & \gamma
\end{array}\right) G=G\left(\begin{array}{cc}
\alpha \gamma & 0 \\
0 & 1
\end{array}\right) G
$$

since one can calculate that

$$
\left(\begin{array}{ll}
\alpha & \beta \\
0 & \gamma
\end{array}\right)=\left(\begin{array}{cc}
0 & 1 \\
-\beta^{-1} & \gamma \beta^{-1}
\end{array}\right)\left(\begin{array}{cc}
\alpha \gamma & 0 \\
0 & 1
\end{array}\right)\left(\begin{array}{ll}
1 & 0 \\
\alpha & \beta
\end{array}\right) \text {. }
$$

So $g_{0}(\alpha \gamma I) \neq 0$. Since $\operatorname{ord}_{\mathbf{p}}(\alpha \gamma)>0$ by construction, this shows $m(g)>0$. If $g_{0}$ vanishes outside of $M_{I}$ it is clear that $m(g)=0$. But if $g_{0}(x) \neq 0$ for some $z \notin M_{I}$, then $\left(\begin{array}{cc}0 & 1 \\ -1 & 0\end{array}\right) \circ z \notin M_{I}$ and $\left(\begin{array}{ll}1 & 1 \\ 0 & 1\end{array}\right) \circ z \notin M_{I}$. Therefore in the process above we are forced to follow the course involving rescaling $\left(\begin{array}{ll}y & x \\ 0 & 1\end{array}\right)$ by $\left(\begin{array}{cc}\pi^{n} & 0 \\ 0 & \pi^{n}\end{array}\right)$ and therefore $m(g)>0$.

The argument now proceeds by induction on $m(g)$. If $m(g)=0$, then $g_{0}$ vanishes outside of $M_{I}$. Let $c=g_{0}(I)$. Then the associated point-pair invariant is

$$
g_{1}(\xi, z)= \begin{cases}c & \text { if } \xi \in M_{z} \\ 0 & \text { otherwise }\end{cases}
$$

so $T_{g} f(z)=\int_{M_{z}} f(\xi) c d \mu(\xi)=c f(z)$. Thus $T_{g}$ acts as a scalar. Assume now that if $m(g) \leq N$ then $T_{g}$ is a polynomial in $T_{\mathbf{p}}$ (of degree $m(g)$ ). In particular if $h_{0}$ is the characteristic function of

$$
G \Delta^{N} G \circ I=\bigcup_{j, \beta}\left(\begin{array}{cc}
\pi^{j} & \beta \\
0 & \pi^{N-j}
\end{array}\right) \circ M_{I},
$$

then $m(h)=N$ so $T_{h}$ is a polynomial in $T_{\mathbf{p}}$ (of degree $N$ ). Moreover,

$$
\begin{aligned}
T_{\mathbf{p}} T_{h} f(z) & =|\pi|_{\mathbf{p}}^{1-k / 2} \int_{H_{\mathbf{p}}} \int_{H_{\mathbf{p}}} f(\xi) h(\xi, \tau) l(\tau, z) d \mu(\xi) d \mu(\tau) \\
& =|\pi|_{\mathbf{p}}^{1-k / 2} \int_{H_{\mathbf{p}}} f(\xi)\left[\int_{H_{\mathbf{p}}} h(\xi, \tau) l(\tau, z) d \mu(\tau)\right] d \mu(\xi)
\end{aligned}
$$

since for any fixed $z$ both $l(\tau, z)$ and $h(\xi, z)$ have compact support. The kernel

$$
k(\xi, z)=|\pi|_{\mathbf{p}}^{1-k / 2} \int_{H_{\mathbf{p}}} h(\xi, \tau) l(\tau, z) d \mu(\tau)
$$

is just $T_{\mathbf{p}} h(\xi, z)$ where $T_{\mathbf{p}}$ acts in the variable $z$. Therefore, by Lemma 9

$$
k(\xi, z)=|\pi|_{\mathbf{p}} \sum_{i=1}^{p^{f}} h\left(\xi, x+\alpha_{i} y+I \pi y\right)+|\pi|_{\mathbf{p}}^{1-k} h\left(\xi, x+I \frac{y}{\pi}\right) .
$$

Now $k_{0}(\xi)=k_{1}(\xi, I)=|1 / v|_{\mathbf{p}}^{k / 2} k(\xi, I)$ so $k_{0}(\xi)=0$ exactly when $k(\xi, I)=$ 0 . Therefore

$$
\begin{aligned}
k_{0}(\xi)=0 & \Leftrightarrow|\pi|_{\mathbf{p}} \sum_{i=1}^{p^{f}}\left|\frac{v}{\pi}\right|_{\mathbf{p}}^{k / 2} h_{1}\left(\xi, \alpha_{i}+I \pi\right)+|\pi|_{\mathbf{p}}^{1-k}\left|\frac{v}{\pi^{-1}}\right|_{\mathbf{p}}^{k / 2} h_{1}\left(\xi, I \pi^{-1}\right)=0 \\
& \Leftrightarrow \sum_{i=1}^{p^{f}} h_{0}\left(\frac{\xi-\alpha_{i}}{\pi}\right)+h_{0}(\xi \pi)=0 .
\end{aligned}
$$


Then we see that $k_{0}\left(I \pi^{m}\right)=0$ if $m>N+1$ and $k_{0}\left(I \pi^{N+1}\right) \neq 0$ since

$$
\begin{aligned}
h_{0}\left(\frac{\pi^{N+1} I}{\pi}\right) & =1, \quad h_{0}\left(\pi^{N+1} I\right)=0, \\
h_{0}\left(\frac{\pi^{N+1} I-\alpha_{i}}{\pi}\right) & =0, \quad \text { for all } \alpha_{i} \neq 0(\bmod \pi) .
\end{aligned}
$$

Thus $m(k)=N+1$. But by construction $T_{k}$ is a polynomial in $T_{\mathbf{p}}$ (of degree $N+1)$. Given any operator $T_{g}$ where $m(g)=N+1$, let $T_{j}=T_{g}-c T_{k}$ where $c=g_{0}\left(I \pi^{N+1}\right) / k_{0}\left(I \pi^{N+1}\right)$. Then $m(j) \leq N$ and hence $T_{j}$ is a polynomial in $T_{\mathbf{p}}$ (of degree $\left.\leq N\right)$. So finally, $T_{g}$ is a polynomial in $T_{\mathbf{p}}$ (of degree $N+1$ ).

The analogy between $T_{\mathrm{p}}$ and the non-Euclidean Laplacian suggests that with a properly defined $L^{2}$ norm, $T_{\mathrm{p}}$ should be a symmetric operator. In fact, that is the case.

Definition. Let $\langle f, g\rangle=\int_{H_{\mathbf{p}}} f(z) \overline{g(z)}|y|_{\mathbf{p}}^{k} d \mu(z)$ whenever this makes sense.

Proposition 12. If $f$ and $g$ are compactly supported locally constant functions on $H_{\mathbf{p}}$, then $\left\langle T_{\mathbf{p}} f, g\right\rangle=\left\langle f, T_{\mathbf{p}} g\right\rangle$.

Proof.

$$
\begin{aligned}
\left\langle T_{\mathbf{p}} f, g\right\rangle & =\int_{H_{\mathbf{p}}} T_{\mathbf{p}} f(z) \overline{g(z)}|y|_{\mathbf{p}}^{k} d \mu(z)=\int_{H_{\mathbf{p}}} \int_{H_{\mathbf{p}}} f(\xi) l(\xi, z) d \mu(\xi) \overline{g(z)}|y|_{\mathbf{p}}^{k} d \mu(z) \\
& =\int_{H_{\mathbf{p}}} f(\xi) \int_{H_{\mathbf{p}}} \overline{g(z)} l(\xi, z)|y|_{\mathbf{p}}^{k} d \mu(z) d \mu(\xi)
\end{aligned}
$$

But

$$
\left\langle f, T_{\mathbf{p}} g\right\rangle=\int_{H_{\mathbf{p}}} f(\xi) \int_{H_{\mathbf{p}}} \overline{g(z)} l(z, \xi)|v|_{\mathbf{p}}^{k} d \mu(z) d \mu(\xi) .
$$

So we must show $l(\xi, z)|y|_{\mathbf{p}}^{k}=l(z, \xi)|v|_{\mathbf{p}}^{k}$. But since $l(\xi, z)=|v / y|_{\mathbf{p}}^{k / 2} l_{1}(\xi, z)$, we are reduced to showing $l_{1}(\xi, z)=l_{1}(z, \xi)$. Since $l_{1}$ is a point-pair invariant, it is enough to show that $l_{1}(\xi, I)=l_{1}(I, \xi)$. Since $\left(\begin{array}{cc}1 & -u \\ 0 & v\end{array}\right) \circ \xi=I$, this is equivalent to $l_{1}(\xi, I)=l_{1}\left(-u v^{-1}+I v^{-1}, I\right)$. This is just saying $u+I v \in G \circ M_{I \pi}$ if and only if $-u v^{-1}+I v^{-1} \in G \circ M_{I \pi}$. By the explicit characterization of this set given above in the definition of $l_{0}$, this is true.

Finally, we note that by breaking $H_{\mathrm{p}}$ up into the $M_{z}$, we are really looking at the tree $G L_{2}\left(K_{\mathrm{p}}\right) / G L_{2}\left(O_{\mathrm{p}}\right)$ (see [Se]). A coset $A G L_{2}\left(O_{\mathrm{p}}\right)$ is identified with $A G L_{2}\left(O_{\mathrm{p}}\right) \circ I=A \circ M_{I}=M_{A \circ I}$.

\section{THE MODULAR GROUPS FOR THE $S$-INTEGERS OF A NUMBER FIELD}

Let $K$ be a number field and $S_{\infty}$ the set of infinite primes of $K$. Let $S$ be any finite set of primes of $K$ containing $S_{\infty}$. We use $S_{\text {fin }}$ to denote $S-S_{\infty}$. Unless noted otherwise, $\mathbf{p}$ will denote an arbitrary prime of $K$, either finite or infinite.

Definition. The set $\left\{x \in K \|\left. x\right|_{\mathbf{p}} \leq 1\right.$ for all $\left.\mathbf{p} \notin S\right\}$ with the operations of addition and multiplication is called the ring of $S$-integers of $K$ and is denoted by $O_{K}(S)$ or simply $O(S)$. 
Definition. The set $\left\{x \in K \|\left. x\right|_{\mathbf{p}}=1\right.$ for all $\left.\mathbf{p} \notin S\right\}$ with the operation of multiplication is called the group of $S$-units of $K$ and is denoted by $U_{K}(S)$ or simply $U(S) . U^{+}(S)$ denotes the subgroup of totally positive $S$-units, i.e., those units all of whose real conjugates are positive.

With this notation the algebraic integers and units of $K$ are specified by taking $S$ to be $S_{\infty}$.

A few words must be said about the ideal structure of $O(S)$. There is a map

$$
\text { Fractional Ideals of } O\left(S_{\infty}\right) \rightarrow \text { Fractional Ideals of } O(S)
$$

given by sending an ideal a to $\mathrm{a} O(S)$, the ideal it generates in $O(S)$. This map is surjective and the kernel is precisely the ideals of $O\left(S_{\infty}\right)$ which are products of only those primes in $S_{\mathrm{fin}}$. The group of ideals of $O(S)$, then, can be identified with the group of ideals of $O\left(S_{\infty}\right)$ modulo the subgroup generated by $S_{\text {fin }}$.

Furthermore, the ideal class group of $O\left(S_{\infty}\right)$ maps onto the ideal class group of $O(S)$. The kernel of this map is precisely those classes containing some ideal that is a product of only primes in $S$. Thus by a judicious choice of $S$ one can ensure that $O(S)$ have class number 1. In general, we use $h(S)$ to denote the class number of the $S$-integers.

To simplify matters so that particular attention can be paid to the finite primes, we will consider only $K$ totally real for the remainder of this chapter. We will, however, make comments on how a more general $K$ could be handled.

Just as one can classically study modular forms attached to the ring of algebraic integers, one should be able to make sense of modular forms attached to the ring of $S$-integers of $K$ for more general $S$. We will consider two different analogs of the modular group.

Definition. $\Gamma_{1}(S)=S L_{2}(O(S))$ is the group of $2 \times 2$ matrices with entries in $O(S)$ and determinant $1 . \Gamma_{U}(S)=G L_{2}(O(S))$ is the group of $2 \times 2$ matrices with entries in $O(S)$ and determinant in $U(S)$.

These groups will act discontinuously on the space which we now define.

Definition. If $\mathbf{p}$ is a real infinite prime, let $H_{\mathbf{p}}=H \cup H^{\prime}=\{x+i y \mid x, y \in \mathbb{R}$, $y \neq 0\}$ be the union of the upper and lower half-plane models of the hyperbolic plane. If $\mathbf{p}$ is a finite prime, let $H_{\mathbf{p}}$ be the p-adic hyperbolic plane as developed in the preceding chapter. Finally, let $H(S)=\prod_{\mathbf{p} \in S} H_{\mathrm{p}}$.

The action of $G L_{2}^{+}(\mathbf{R})$ on the upper half-plane $H$ via fractional linear transformations is well known. Because we will not always be requiring that our matrices have totally positive determinant, it is necessary to include a lower half-plane as well. Thus with the results of the preceding section, for all $\mathbf{p}$ there is an action of $G L_{2}\left(K_{\mathrm{p}}\right)$ on $H_{\mathrm{p}}$.

This gives rise to an action of $\Gamma_{U}(S)$ on $H(S)$ by: If $A \in \Gamma_{U}(S), z \in H(S)$, $z=\left(\ldots, z_{\mathbf{p}}, \ldots\right)$, then $A \circ z=\left(\ldots, A_{\mathbf{p}} \circ z_{\mathbf{p}}, \ldots\right)$ where $A_{\mathbf{p}}$ means the $\mathbf{p}-$ conjugate of $A$ (obtained by taking the conjugate of each entry of $A$ ) if $\mathrm{p}$ is infinite, and simply $A$ viewed as an element of $G L_{2}\left(K_{\mathbf{p}}\right)$ if $\mathbf{p}$ is finite.

If we were to consider $K$ with complex primes $\mathbf{p}$, then we would let $H_{\mathbf{p}}=$ $H^{3}=\{x+k y \mid x \in \mathbf{C}, y \in \mathbf{R}, y>0\}$ be hyperbolic 3-space, viewed as a subset of the quaternions. Then $S L_{2}(\mathbf{C})$ acts on $H_{\mathbf{p}}$ for complex $\mathbf{p}$ via fractional 
linear transformations (cf. [St]). The only problem with this is that then we would be able only to consider matrices of determinant one (or at most matrices whose determinants were real and positive in each complex conjugate). If one is willing to accept this then much of what follows can be trivially modified to that situation. That course is not followed here since we wish to show in $\S$ VII the connections to Herrmann's theory for Hilbert modular forms, and since as $S$ is made larger we will find the fundamental domain of $\Gamma_{U}(S)$ changes more nicely than that of $\Gamma_{1}(S)$.

A point $z \in H(S)$ will be denoted by $x+I y$ where $x=\left(\ldots, x\left(z_{\mathrm{p}}\right), \ldots\right)$ and $I y=\left(\ldots, I_{\mathrm{p}} y\left(z_{\mathrm{p}}\right), \ldots\right)$ and $I_{\mathrm{p}}$ means $i, k$, or the $I_{\mathrm{p}}$ of $\S I$ respectively as $\mathbf{p}$ is real, complex, or finite. $z_{\text {inf }}$ and $z_{\text {fin }}$ will be used to denote the obvious infinite and finite parts of the vector $z$.

For each $\mathbf{p} \in S_{\mathrm{fn}}, L_{\mathbf{p}}$ denotes the quadratic extension of $K_{\mathbf{p}}$ used in constructing $H_{\mathrm{p}}$. Similarly, let $L_{\mathrm{p}}$ denote the complex numbers (or quaternions) as $\mathbf{p}$ is real (or complex).

As in [St] one sees that $\Gamma_{U}(S)$ and $\Gamma_{1}(S)$ both act discontinuously on $H(S)$ and thus have fundamental domains. When $S=S_{\infty}$ the determination of an explicit fundamental domain for these groups is a classical problem. One would not expect it to be a simpler process for arbitrary $S$. However, it is at least no more difficult as we will see.

The approach we use here is very concrete. We reduce the question of determining the fundamental domain to that of determining the fundamental domains for certain groups acting on $H\left(S_{\infty}\right)$. This particular form of the domain will be needed later, though it also helps clarify the nature of the groups $\Gamma_{U}(S)$ and $\Gamma_{1}(S)$.

We first need some notation in order to study the action of these groups on $H\left(S_{\text {fin }}\right)$.

Definition. For any point $w=\left(\ldots, w_{\mathbf{p}}, \ldots\right) \in H\left(S_{\mathrm{fin}}\right)$, let $\boldsymbol{M}_{w}=\prod_{\mathrm{p} \in S_{\mathrm{fn}}} \boldsymbol{M}_{w_{\mathbf{p}}}$, where $M_{w_{\mathbf{p}}} \subset H_{\mathbf{p}}$ is the neighborhood of $w_{\mathbf{p}}$ defined in $\S \mathbf{I}$.

By Proposition 5 of $\S I$, the action of any $A \in G L_{2}(K)$ on $M_{w}$ is just $A \circ M_{w}=$ $M_{A \circ w}$.

We first consider investigating a fundamental domain for $\Gamma_{U}(S)$. Temporarily, let $G$ be the subgroup of the ideal group of $O\left(S_{\infty}\right)$ generated by those primes in $S_{\text {fin }}$. Let $H \subset G$ be the subgroup of principle ideals in $G$. Then $C_{S}=G / H$ is a subgroup of the ideal class group of $O\left(S_{\infty}\right)$. Consider $C_{S} / C_{S}^{2}$, i.e., $C_{S}$ modulo squares in $C_{S}$. Then a finite set $\left\{\mathbf{a}_{i}\right\}$ of ideals of $G$ can be found giving a complete set of representatives of $C_{S} / C_{S}^{2}$. Fix any such set $\left\{\mathbf{a}_{i}\right\}$.

Definition. If a is an ideal of $O\left(S_{\infty}\right)$ with all its prime factors in $S$, let $w(\mathbf{a}) \in$ $H\left(S_{\text {fin }}\right)$ be the point $\left(\ldots, I_{\mathbf{p}} \pi^{n_{\mathbf{p}}}, \ldots\right)$ where $n_{\mathbf{p}}=\operatorname{ord}_{\mathbf{p}}(\mathbf{a})$. Conversely, if $I y=\left(\ldots, I_{\mathrm{p}} y_{\mathrm{p}}, \ldots\right) \in H\left(S_{\mathrm{fin}}\right)$, let $\operatorname{Id}(I y)$ be the ideal $\prod_{\mathrm{p} \in S_{\mathrm{fn}}} \mathbf{p}^{\operatorname{ord}_{\mathrm{p}}\left(y_{\mathrm{p}}\right)}$. Thus $\operatorname{Id}(w(\mathbf{a}))=\mathbf{a}$, but $w(\operatorname{Id}(I y))$ is $I y$ only "up to units".

Lemma 1. If $w \in H\left(S_{\mathrm{fin}}\right)$, then there exists an $A \in \Gamma_{U}(S)$ such that $A \circ M_{w}=$ $M_{w\left(\mathbf{a}_{i}\right)}$ for some $i$. Furthermore, $i$ is uniquely determined.

Proof. Given $w \in H\left(S_{\mathrm{fn}}\right)$, write $w=x+I y=\left(\ldots, x_{\mathrm{p}}, \ldots\right)+\left(\ldots, I_{\mathrm{p}} y_{\mathrm{p}}, \ldots\right)$. By an application of the Chinese Remainder Theorem, a $t \in O(S)$ can be found such that $x_{\mathbf{p}}-t \in O_{\mathbf{p}} y_{\mathbf{p}}$ for all $\mathbf{p} \in S_{\mathrm{fin}}$. Therefore $\left(\begin{array}{cc}1 & -t \\ 0 & 1\end{array}\right) \circ M_{w}=M_{I y}$. 
The lemma is now reduced to showing: $A \circ M_{I y}=M_{I v}$ for some $A \in \Gamma_{U}(S)$ if and only if $\operatorname{Id}(I y)$ and $\operatorname{Id}(I v)$ have the same image in $C_{S} / C_{S}^{2}$, (i.e., if and only if $\operatorname{Id}(I v)=(\alpha) \mathbf{a}^{2} \operatorname{Id}(I y)$ for some ideal a and number $\alpha \in K$, both divisible only by primes in $\left.S_{\mathrm{fin}}\right)$.

First, suppose $A \in \Gamma_{U}(S)$ and $A \circ M_{I y}=M_{I v}$. Then $A \circ M_{I_{\mathrm{p}} y_{\mathrm{p}}}=M_{I_{\mathrm{p}} v_{\mathrm{p}}}$ for each $\mathbf{p} \in S_{\mathrm{fin}}$. If $l=\operatorname{ord}_{\mathbf{p}}\left(y_{\mathbf{p}}\right)$ and $m=\operatorname{ord}_{\mathbf{p}}\left(v_{\mathbf{p}}\right)$, then

$$
\left(\begin{array}{cc}
\pi^{-m} & 0 \\
0 & 1
\end{array}\right) A\left(\begin{array}{cc}
\pi^{l} & 0 \\
0 & 1
\end{array}\right) \circ M_{I_{\mathrm{p}}}=M_{I_{\mathrm{p}}}
$$

so by Corollary 4 of $\S I$

$$
\left(\begin{array}{cc}
\pi^{-m} & 0 \\
0 & 1
\end{array}\right) A\left(\begin{array}{cc}
\pi^{l} & 0 \\
0 & 1
\end{array}\right)\left(\begin{array}{cc}
\pi^{n} & 0 \\
0 & \pi^{n}
\end{array}\right) \in G L_{2}\left(O_{\mathbf{p}}\right)
$$

for some integer $n$. Taking determinants shows $\pi^{2 n+l-m} \operatorname{det}(A) \in U_{\mathbf{p}}$, or more suggestively $\left(\pi^{m}\right)=(\operatorname{det}(A))\left(\pi^{n}\right)^{2}\left(\pi^{l}\right)$ in $K_{\mathrm{p}}$. Combining this local information for each $\mathbf{p} \in S_{\text {fin }}$ shows $\operatorname{Id}(I v)=(\operatorname{det}(A)) \mathbf{a}^{2} \operatorname{Id}(I y)$ for some ideal a .

Conversely, suppose $\operatorname{Id}(I v)=(\alpha) \mathbf{a}^{2} \operatorname{Id}(I y)$ and choose any nonzero $S$-integer $d \in \mathbf{a}^{-1}$. Then we can find an $S$-integer $c$ such that $(c, d)=(1)$ in $O(S)$ and $\operatorname{ord}_{\mathbf{p}}\left(c I_{\mathbf{p}} y_{\mathbf{p}}+d\right)=\operatorname{ord}_{\mathbf{p}}\left(\mathbf{a}^{-1}\right)$ for all $\mathbf{p} \in S_{\mathrm{fin}}$. Indeed, this just places congruence conditions on $c$ for $\mathbf{p} \in S_{\mathrm{fin}}$ and for any additional $\mathbf{p}$ dividing $(d)$. By the Chinese Remainder Theorem these finitely many conditions can be met.

Now $S$-integers $a$ and $b$ can be chosen so that $A=\left(\begin{array}{ll}a & b \\ c & d\end{array}\right)$ has determinant $\alpha$. Thus $A \in \Gamma_{U}(S)$ and we calculate

$$
A \circ I_{\mathbf{p}} y_{\mathbf{p}}=u_{\mathbf{p}}+\frac{\operatorname{det}(A) I_{\mathbf{p}} y_{\mathbf{p}}}{\mathbf{N}_{K_{\mathbf{p}}}^{L_{\mathbf{p}}}\left(c I_{\mathbf{p}} y_{\mathbf{p}}+d\right)} \quad \text { for some } u_{\mathbf{p}} \in K_{\mathbf{p}}
$$

But

$$
\operatorname{ord}_{\mathbf{p}}\left(\frac{\operatorname{det}(A) I_{\mathbf{p}} y_{\mathbf{p}}}{N_{K_{\mathbf{p}}}^{L_{\mathbf{p}}}\left(c I_{\mathbf{p}} y_{\mathbf{p}}+d\right)}\right)=\operatorname{ord}_{\mathbf{p}}\left(\frac{(\alpha) \operatorname{Id}(I y)}{\mathbf{a}^{-2}}\right)=\operatorname{ord}_{\mathbf{p}}(\operatorname{Id}(I v))
$$

by construction. So $A \circ M_{I_{\mathbf{p}} y_{\mathbf{p}}}=M_{u_{\mathrm{p}}+I_{\mathbf{p}} v_{\mathbf{p}}}$ for each $\mathbf{p} \in S_{\text {fin }}$, and some $u_{\mathbf{p}}$. Now replacing $A$ by $\left(\begin{array}{cc}1 & -t \\ 0 & 1\end{array}\right) \circ A$ for appropriate $t$ (chosen similarly to that at the beginning of the proof), we have $A \circ M_{I_{\mathrm{p}} y_{\mathrm{p}}}=M_{I_{\mathrm{p}} v_{\mathrm{p}}}$ for all $\mathbf{p} \in S_{\mathrm{fin}}$. Thus $A \circ M_{I y}=M_{I v}$.

Proposition 2. A fundamental domain $F$ for the action of $\Gamma_{U}(S)$ on $H(S)$ of the following form exists:

With $\left\{\mathbf{a}_{i}\right\}$ as above, let $F_{\infty, i} \subset H\left(S_{\infty}\right)$ be a fundamental domain for

$$
\begin{array}{r}
\Gamma_{\mathbf{a}_{i}}=\left\{\left(\begin{array}{ll}
a & b \\
c & d
\end{array}\right) \in \Gamma_{U}(S) \mid c \in \mathbf{b a}_{i}^{-1}, b \in \mathbf{b a}_{i} a, d \in \mathbf{b},\right. \\
\left.(a d-b c)=\mathbf{b}^{2} \text { for some ideal } \mathbf{b} \text { of } O\left(S_{\infty}\right)\right\} .
\end{array}
$$

Let $F_{i}=F_{\infty, i} \times M_{w\left(\mathbf{a}_{i}\right)} \subset H(S)$. Then $F=\bigcup_{i} F_{i}$.

Proof. Given any point $z \in H(S)$, by Lemma 1 it is $\Gamma_{U}(S)$-equivalent to points whose finite parts are in $M_{w\left(a_{i}\right)}$ for exactly one $i$. Suppose $z$ and $A \circ z$ have 
finite parts in $M_{w\left(\mathbf{a}_{i}\right)}$ for some $A \in \Gamma_{U}(S)$. Then $A \circ M_{w\left(\mathbf{a}_{i}\right)}=M_{w\left(\mathbf{a}_{i}\right)}$, so $A \circ M_{I_{\mathrm{p}} \pi^{m}}=M_{I_{\mathrm{p}} \pi^{m}}$ where $m=\operatorname{ord}_{\mathbf{p}}\left(\mathbf{a}_{i}\right)$. Thus

$$
\left(\begin{array}{cc}
\pi^{-m} & 0 \\
0 & 1
\end{array}\right) A\left(\begin{array}{cc}
\pi^{m} & 0 \\
0 & 1
\end{array}\right) \circ M_{I_{\mathrm{p}}}=M_{I_{\mathrm{p}}}
$$

so by Corollary 4 of $\S I$,

$$
\left(\begin{array}{cc}
\pi^{-m} & 0 \\
0 & 1
\end{array}\right) A\left(\begin{array}{cc}
\pi^{m} & 0 \\
0 & 1
\end{array}\right)=\left(\begin{array}{cc}
\pi^{n} & 0 \\
0 & \pi^{n}
\end{array}\right) B
$$

for some $B \in G L_{2}\left(O_{\mathbf{p}}\right)$. If $B=\left(\begin{array}{ll}e & f \\ g & h\end{array}\right)$ then

$$
A=\left(\begin{array}{cc}
e \pi^{n} & f \pi^{n+m} \\
g \pi^{n-m} & h \pi^{n}
\end{array}\right)
$$

Combining this local information for each $\mathbf{p} \in S_{\mathrm{fin}}$ we find $A$ is in the group described in the statement of the proposition. Thus by the definition of $F_{\infty, i}$, no two points of $F_{i}$ are $\Gamma_{U}(S)$-equivalent and so $F$ is a fundamental domain.

An explicit determination of possible $F_{\infty, i}$ will be left unaddressed here, as that only involves the infinite primes.

Before we turn to $\Gamma_{1}(S)$, we note that if $K$ is a quadratic field and $S$ is sufficiently large then the set $\left\{\mathbf{a}_{i}\right\}$ is a set of representatives of the (weak) genus classes of $K$.

We now consider $\Gamma_{1}(S)$. Temporarily, let $G$ be the subgroup of the ideal group of $O\left(S_{\infty}\right)$ generated by those primes in $S_{\mathrm{fin}}$. Fix any $\left\{\mathbf{b}_{i}\right\}$, a complete set of representatives of $G / G^{2}$. Then

Lemma 3. If $w \in H\left(S_{\mathrm{fin}}\right)$ then there exists an $A \in \Gamma_{1}(S)$ such that $A \circ M_{w}=$ $M_{w\left(\mathbf{b}_{i}\right)}$ for some $i$. Furthermore $i$ is uniquely determined.

Proof. If we try to repeat the argument of Lemma 1 everything goes through as before except we are restricting the determinant of the matrices to be 1 .

The analog of Proposition 2 is

Proposition 4. A fundamental domain $F$ for the action of $\Gamma_{1}(S)$ on $H(S)$ of the following form exists. With $\mathbf{b}_{i}$ as above, let $F_{\infty, i} \subset H\left(S_{\infty}\right)$ be a fundamental domain for

$$
\Gamma_{1, \mathbf{b}_{i}}=\left\{\left(\begin{array}{ll}
a & b \\
c & d
\end{array}\right) \in \Gamma_{1}(S) \mid c \in \mathbf{b}_{i}^{-1}, b \in \mathbf{b}_{i}, a, d \in O\left(S_{\infty}\right)\right\} .
$$

Let $F_{i}=F_{\infty, i} \times M_{w\left(\mathbf{b}_{i}\right)} \subset H(S)$. Then $F=\bigcup_{i} F_{i}$.

Proof. Exactly as Proposition 2, taking into account that we only allow determinant 1 matrices.

Notice that as $S$ grows, the fundamental domain of $\Gamma_{1}(S)$ grows more complicated in its finite components, but that once $S$ contains enough primes to generate the ideal class group, that of $\Gamma_{U}(S)$ stays the same in its finite components.

It is well known that $\Gamma_{1}\left(S_{\infty}\right)$ has $h\left(S_{\infty}\right)$ cusps. We will see that the analogous result holds for $\Gamma_{U}(S)$ and $\Gamma_{1}(S)$. Although the argument is identical to the classical one, it is included here for completeness. 
Definition. A matrix $A \in G L_{2}(K)$ is called parabolic if $A$ has Jordan form $\left(\begin{array}{ll}\lambda & 1 \\ 0 & \lambda\end{array}\right)$; i.e., if $B A B^{-1}=\left(\begin{array}{ll}\lambda & 1 \\ 0 & \lambda\end{array}\right)$ for some $B \in G L_{2}(\bar{K})$ where $\bar{K}$ is the algebraic closure of $K$.

The parabolic matrices are those that produce what is essentially a translation when applied to $H(S)$ and thus are necessary if one is to develop Fourier series.

A simple calculation gives

Lemma 5. If $A \in G L_{2}(K)$ acts nontrivially on $H(S)$, then $A$ is parabolic if and only if $\operatorname{det}(A)=(\operatorname{Tr}(A) / 2)^{2}$.

Lemma 6. If $A \in G L_{2}(K)$ is parabolic, then $A$ acting on $\prod_{\mathbf{p} \in S}\left(L_{\mathbf{p}} \cup\{\infty\}\right)$ via fractional linear transformations has exactly one fuxed point, and that lies in $K \cup\{\infty\}$ (embedded along the diagonal).

Proof. Let $A=\left(\begin{array}{ll}a & b \\ c & d\end{array}\right)$. Then if $c=0$, then $A$ is parabolic means $a=d$ and $b \neq 0$. Thus $a \circ z=z$ means $a z+b=a z$, which can happen only if $z=\infty$.

If $c \neq 0$, then $A \circ z=z$ means $a z+b=c z^{2}+d z$ or $z$ is a root of $c x^{2}+(d-a) x-b=0$. Therefore

$$
\begin{aligned}
z & =\frac{a-d \pm \sqrt{(d-a)^{2}+4 c b}}{2 c}=\frac{a-d \pm \sqrt{(d+a)^{2}-4(a d-b c)}}{2 c} \\
& =\frac{a-d \pm \sqrt{\operatorname{Tr}(A)^{2}-4 \operatorname{det}(A)}}{2 c}=\frac{a-d}{2 c}
\end{aligned}
$$

by the preceding lemma. So $z \in K$.

Thus we can associate to any parabolic matrix $A$ its fixed point in $K \cup\{\infty\}$. One can also check that two parabolic matrices commute if and only if they have the same fixed point. Classifying parabolic elements by fixed points is too broad for our purposes, however. We need to treat $A$ and $B A B^{-1}$, for any $B \in \Gamma_{U}(S)$, the same since (once modular forms are defined) a Fourier series associated to $A$ and one associated to $B A B^{-1}$ would be the same. Since if $A$ fixes $z$ then $B A B^{-1}$ fixes $B \circ z$, we are led to put

Definition. The cusps of a subgroup $G \subset G L_{2}(K)$ are the orbits of elements of $K \cup\{\infty\}$ under the action of $G$.

Proposition 7. $\Gamma_{U}(S)$ and $\Gamma_{1}(S)$ have $h(S)$ cusps.

Proof. Let $\alpha \in K \cup\{\infty\}$. Write $\alpha=r / s$ where $r, s \in O(S)$. (Take $s=0$, $r \neq 0$ to represent $\infty$.) We can then associate to $\alpha$ the $O(S)$ ideal $\mathbf{a}=$ $(r, s)$. If we chose to write $\alpha=t / u, t, u \in O(S)$, instead, then $r / s=t / u$ so $(t)(r, s)=(t r, t s)=(t r, u r)=(r)(t, u)$. Therefore the ideal class of a is at least completely determined, so we associate to $\alpha$ that class. Since every ideal is generated by two elements, each class corresponds to at least one $\alpha$.

Now let $\alpha, \beta \in K \cup\{\infty\}$. Write $\alpha=r / s$ and $\beta=t / u$ with $r, s, t, u \in$ $O(S)$. We will show $\alpha$ and $\beta$ are in the same orbit under either $\Gamma_{U}(S)$ or $\Gamma_{1}(S)$, if and only if $(r, s)$ and $(t, u)$ are in the same ideal class of $O(S)$.

First let $B=\left(\begin{array}{ll}a & b \\ c & d\end{array}\right) \in \Gamma_{U}(S)$ and suppose $B \circ \alpha=\beta$. Then $\left(\begin{array}{ll}a & b \\ c & d\end{array}\right) \circ(r / s)=$ $(t / u)$ implies that

$$
\left(\begin{array}{ll}
a & b \\
c & d
\end{array}\right)\left(\begin{array}{l}
r \\
s
\end{array}\right)=\lambda\left(\begin{array}{l}
t \\
u
\end{array}\right) \text { for some } \lambda \in K
$$


So $(\lambda)(t, u)=(a r+b s, c r+d s)$. But since $B$ is invertible, then $(a r+b s, c r+$ $d s)=(r, s)$ as ideals in $O(S)$. Therefore $(t, u)$ and $(r, s)$ are in the same ideal class.

Conversely, if $(r, s)$ and $(t, u)$ are in the same ideal class of $O(S)$, then multiplying through by elements of $O(S)$ if necessary we might as well assume that $\alpha=r / s, \beta=t / u$, and $(r, s)=(t, u)=\mathbf{a}$. But then elements $e, f, g, h$ of the $O(S)$ ideal $\mathbf{a}^{-1}$ can be found such that $e r-f s=1$ and $g t-h u=1$. Then

$$
\left(\begin{array}{ll}
t & h \\
u & g
\end{array}\right)\left(\begin{array}{ll}
r & f \\
s & e
\end{array}\right)^{-1} \circ \frac{r}{s}=\frac{t}{u}
$$

and one checks that

$$
\left(\begin{array}{ll}
t & h \\
u & g
\end{array}\right)\left(\begin{array}{ll}
r & f \\
s & e
\end{array}\right)^{-1} \in \Gamma_{1}(S)
$$

A full understanding of the cusps requires the explicit construction of a fundamental domain for the action of $\Gamma_{\mathbf{a}_{i}}$ and $\Gamma_{1, \mathbf{b}_{i}}$ on $H\left(S_{\infty}\right)$, which can be done as in [Si]. These groups are readily seen to have $h\left(S_{\infty}\right)$ cusps themselves. Each (representative of a) cusp (as defined here) is indeed fixed by some parabolic elements of even the group $S L_{2}\left(O\left(S_{\infty}\right)\right)$. Furthermore, following the discussion of [Si], fundamental domains can be constructed that are bounded except for pieces stretching off to one representative of each cusp.

Proceeding from $[\mathrm{H}]$ where the same groups naturally arise, Gundlach $[\mathrm{G} 1$, G2] investigated Hilbert modular forms for the groups $\Gamma_{1, \mathbf{b}_{i}}$. In fact, he developed a rather full theory of Eisenstein series and Poincare series, including estimates on the dimension of the space of cusp forms of given weight.

\section{MODULAR FORMS}

Following Stark we set

Definition. The $S$-norm of a vector $z=\left(\ldots, z_{\mathbf{p}}, \ldots\right) \in \prod_{\mathbf{p} \in S} L_{\mathbf{p}}$ is

$$
\mathbf{N}_{S}(x)=\prod_{\mathbf{p} \text { real }} z_{\mathbf{p}} \prod_{\mathbf{p} \in S \text { not real }}\left|z_{\mathbf{p}}\right|_{\mathbf{p}}
$$

(If we considered complex primes, then here $\left|z_{\mathbf{p}}\right|_{\mathbf{p}}$ for $\mathbf{p}$ complex would denote the quaternionic absolute value squared,

$$
\left.\left|x_{1}+x_{2} i+x_{3} j+x_{4} k\right|_{\mathbf{p}}=x_{1}^{2}+x_{2}^{2}+x_{3}^{2}+x_{4}^{2} .\right)
$$

In particular, if $x \in K$ is viewed as a vector in $\prod_{\mathbf{p} \in S} L_{\mathbf{p}}$, then $\mathbf{N}_{S}(z) \in \mathbf{Z}$ if $z$ is an $S$-integer and $\mathbf{N}_{S}(z)= \pm 1$ if $z$ is an $S$-unit.

Definition. A function $f$ on $H\left(S_{\text {fin }}\right)$ will be called locally constant if it is constant on all sets of the form $M_{w}$ for $w \in H\left(S_{\text {fin }}\right)$.

Definition. The weight $k$-slash operator on functions $f$ defined on $H(S)$ is given by

$$
\left.f\right|_{S, k} A(z)=\left.f\right|_{k} A(z)=\mathbf{N}_{S}(\operatorname{det}(A))^{k / 2} \mathbf{N}_{S}(c z+d)^{-k} f(A \circ z)
$$

for any $A=\left(\begin{array}{ll}a & b \\ c & d\end{array}\right) \in G L_{2}(K)$. 
If $k$ is odd then we must fix some choice of the square root, say the principal value.

This operator is just the product of the local slash operators of $\S I$ for each finite prime in $S$ and the obvious local slash operators for the infinite primes.

We also recall the weight $k$ Laplacians for the real infinite primes.

Definition. If $\mathbf{p}$ is real prime and $z_{\mathbf{p}}=x+i y$, let

$$
\Delta_{\mathbf{p}, k}=-y^{2}\left(\frac{\partial^{2}}{\partial x^{2}}+\frac{\partial^{2}}{\partial y^{2}}\right)+i k y\left(\frac{\partial}{\partial x}+i \frac{\partial}{\partial y}\right) .
$$

This is the fundamental differential operator commuting with the local weight $k$ slash operator, and is the model after which $T_{\mathbf{p}, k}$ was constructed for finite primes in $\S$ I. Thus $\Delta_{\mathbf{p}, k}$ and $T_{\mathbf{p}, k}$ commute with the $S$-slash operator as well.

By using vector forms as in [Sr], complex primes can be handled, though things become a bit messier. Alternately, by restricting to weight 0 some of the difficulties are circumvented and the following material goes through in essence. One need only define a weight 0 Laplacian

$$
\Delta_{\mathbf{p}, 0}=-y^{2}\left(\frac{\partial^{2}}{\partial x_{1}^{2}}+\frac{\partial^{2}}{\partial x_{2}^{2}}+\frac{\partial^{2}}{\partial y^{2}}\right)+y \frac{\partial}{\partial y}
$$

for $\mathbf{p}$ complex, $z_{\mathbf{p}}=x_{1}+i x_{2}+k y$ as in [Sa].

We restrict our attention here to cusp forms in order to focus on the new aspects of the theory introduced by the p-adic planes.

Definition. If $K$ is a totally real number field, then an $S$-modular cusp form of weight $k$ and eigenvalue system $\Lambda$ for $\Gamma_{1}(S)$, where $\Lambda=\left\{\lambda_{\mathbf{p}}\right\}_{\mathbf{p} \in S}$ and $\lambda_{\mathbf{p}} \in \mathbf{C}$, is a function $f: H(S) \rightarrow \mathbf{C}$ satisfying

(1) $\left.f\right|_{k} A=f$ for all $A \in \Gamma_{1}(S)$.

(2) If $\alpha \in K$ is a (representative of a) cusp of $\Gamma_{1}(S)$, and $A \in G L_{2}(K)$ is such that $\alpha=A \circ \infty$, then $\mathbf{N}_{S_{\infty}}(y)^{k / 2}\left(\left.f\right|_{k} A\right)(z) \rightarrow 0$ as $\left|\mathbf{N}_{S_{\infty}}(y)\right| \rightarrow \infty$.

(3a) $\Delta_{\mathbf{p}, k} f=\lambda_{\mathbf{p}} f$ for all $\mathbf{p} \in S_{\infty}$.

(3b) $f$ is locally constant on $H\left(S_{\text {fin }}\right)$ and $T_{\mathbf{p}, k} f=\lambda_{\mathbf{p}} f$ for all $\mathbf{p} \in S_{\text {fin }}$.

We denote the complex vector space of such weight- $k$ cusp forms by $\mathrm{S}_{k}\left(\Gamma_{1}(S), \Lambda\right)$.

Since $\left(\begin{array}{cc}-1 & 0 \\ 0 & -1\end{array}\right) \in \Gamma_{1}(S)$, condition (1) says that in particular

$$
\mathbf{N}_{S}(-1)^{k} f(z)=\left.f\right|_{k}\left(\begin{array}{cc}
-1 & 0 \\
0 & -1
\end{array}\right)(z)=f(z)
$$

Since $\mathbf{N}_{S}(-1)=(-1)^{n}$ where $n=[K: \mathbf{Q}]$ for a totally real field, then $n k$ must be an even integer if any nonzero forms are to exist.

If $f \in \mathbf{S}_{k}\left(\Gamma_{1}(S), \Lambda\right)$, then actually $\left.f\right|_{k} A=f$ for any $A \in \Gamma_{\left(U^{+}\right)^{2}}(S)=$ $\left\{A \in G L_{2}(O(S)) \mid \operatorname{det}(A) \in U^{+}(S)^{2}\right\}$. This is simply because by the definition of the slash operator $\left.f\right|_{k}\left(\begin{array}{cc}\varepsilon & 0 \\ 0 & \varepsilon\end{array}\right)=f$ for any $\varepsilon \in U^{+}(S)$. But then, since $\Gamma_{U}(S) / \Gamma_{\left(U^{+}\right)^{2}}(S)$ is a finite abelian group (isomorphic to $U(S) / U^{+}(S)^{2}$ under the determinant map), we can decompose

$$
\mathbf{S}_{k}\left(\Gamma_{1}(S), \Lambda\right)=\bigoplus_{\chi} \mathbf{S}_{k}\left(\Gamma_{U}(S), \chi, \Lambda\right)
$$


where the sum is taken over all characters $\chi$ of $U(S) / U^{+}(S)^{2}$ and $f \in$ $\mathbf{S}_{k}\left(\Gamma_{U}(S), \chi, \Lambda\right)$ means $f \in \mathbf{S}_{k}\left(\Gamma_{1}(S), \Lambda\right)$ and $\left.f\right|_{k} A=\chi(\operatorname{det}(A)) f$ for any $A \in \Gamma_{U}(S)$. If $k$ is even then actually $\left.f\right|_{k}\left(\begin{array}{cc}\varepsilon & 0 \\ 0 & \varepsilon\end{array}\right)=f$ for any $\varepsilon \in U(S)$, so that $\chi$ must be trivial on $U(S)^{2}$.

Recall from the theory of partial differential operators that condition (3a) implies that $f$ is smooth in $z_{\mathbf{p}}$ for each infinite $\mathbf{p}$.

If $f \in \mathbf{S}_{k}\left(\Gamma_{1}(S), \Lambda\right)$, then $\left.f\right|_{k}\left(\begin{array}{ll}1 & t \\ 0 & 1\end{array}\right)(z)=f(z+t)=f(z)$ for any $t \in O(S)$. Thus $f$ should have a Fourier expansion at the cusp $\infty$. In fact, it should have a Fourier expansion at every cusp. Since by taking $S$ large enough we can ensure that $h(S)=1$ so that there is only one cusp, we will omit considering the more general multiple cusps. The modifications usually used in more classical settings to handle cusps other than $\infty$ can all be carried out here easily.

We must recall some facts from harmonic analysis on the spaces we are dealing with. The following definitions and proposition can be found in Tate's thesis [T].

If $\mathbf{p}$ is a prime of $K$ and $K_{\mathbf{p}}$ the completion of $K$ with respect to $\mathbf{p}$, define the additive homomorphism $\Phi_{\mathrm{p}}: K_{\mathrm{p}} \rightarrow \mathbf{R} / \mathrm{Z}$ so that $\Phi_{\mathrm{p}}$ is the composition of the sequence of maps

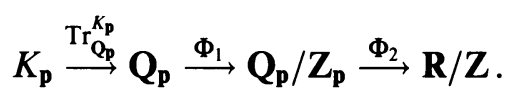

Here $\mathbf{Q}_{\mathbf{p}}$ is the completion of the rationals with respect to the rational prime $\mathbf{p}$ (possible $\infty$ ) over which $\mathbf{p}$ lies and $\Phi_{1}$ is the obvious quotient map. If $\mathbf{p}$ is infinite, then $\Phi_{2}$ is the identity, while if $\mathbf{p}$ is finite, then $\Phi_{2}$ sends an element of $\mathbf{Q}_{\mathbf{p}} / \mathbf{Z}_{\mathbf{p}} \simeq \mathbf{Q} / \mathbf{Z}$ into $\mathbf{R} / \mathbf{Z}$ via first multiplying by -1 and then utilizing the inclusion of $\mathbf{Q}$ into $\mathbf{R}$.

Proposition 1. The additive group $K_{\mathbf{p}}$ is self-dual under the identification of $\nu \in$ $K_{\mathbf{p}}$ with the character $x \mapsto \exp \left(2 \pi i \Phi_{\mathbf{p}}(\nu x)\right)$. Furthermore, for finite $\mathbf{p}$, this character is trivial on $O_{\mathbf{p}}$ if and only if $\nu \in \delta_{\mathbf{p}}^{-1}$, the local inverse different.

(We will use $\delta_{\mathrm{p}}$ to mean both an element of $K_{\mathrm{p}}$ and the ideal it generates.) Now we piece these together to study $O(S)$.

Definition. The $S$-trace of a vector $x \in \prod_{\mathbf{p} \in S} K_{\mathbf{p}}$ is $\operatorname{Tr}_{S}(x)=\sum_{\mathbf{p} \in S} \Phi_{\mathbf{p}}\left(x_{\mathbf{p}}\right) \in$ $\mathbf{R} / \mathbf{Z}$.

If we define multiplication of two vectors $\nu=\left(\ldots, \nu_{\mathrm{p}}, \ldots\right)$ and $x=$ $\left(\ldots, z_{\mathbf{p}}, \ldots\right)$ in $\prod_{\mathbf{p} \in S} K_{\mathbf{p}}$ by $\nu x=\left(\ldots, \nu_{\mathbf{p}} x_{\mathbf{p}}, \ldots\right)$, then

Proposition 2. The additive group $\prod_{\mathbf{p} \in S} K_{\mathbf{p}}$ is self-dual under the identification of a vector $\nu \in \prod_{\mathbf{p} \in S} K_{\mathbf{p}}$ with the character $x \mapsto \exp \left(2 \pi i \operatorname{Tr}_{S}(\nu x)\right)$.

It is natural to introduce

Definition. The $S$-different $\delta(S)$ is the $O(S)$-ideal defined by

$$
\delta(S)^{-1}=\left\{x \in K \mid \operatorname{Tr}_{S}(x \alpha)=0 \text { for all } \alpha \in O(S)\right\} .
$$

That is, the inverse $S$-different is the dual module of $O(S)$ with respect to the $S$-trace. Note that $\delta\left(S_{\infty}\right)$ is just the usual global different. $\delta(S)$ is also easily identified by 
Proposition 3. $\delta(S)$ is the ideal generated by $\delta\left(S_{\infty}\right)$ in $O(S)$.

Proof. Recall (cf. [La]) that for any $x \in K$

$$
\operatorname{Tr}_{\mathbf{Q}}^{K}(x)=\sum_{\mathbf{p} \mid p} \operatorname{Tr}_{\mathbf{Q}_{\mathbf{p}}}^{K_{\mathbf{p}}}(x)
$$

in $\mathbf{Q}_{\mathbf{p}}$ for any rational prime $p$. Then considering the partial fraction decomposition of the rational number $\operatorname{Tr}_{\mathbf{Q}}^{K}(x)$ as $a_{0}+\sum_{p} a_{p} / p^{n_{p}}$ where $0 \leq a_{p}<p^{n_{p}}$ and $a_{i} \in \mathbf{Z}$, this means

$$
\operatorname{Tr}_{S_{\infty}}(x)+\sum_{\mathbf{p} \text { finite }} \Phi_{\mathbf{p}}(x)=0 .
$$

Let $x \in \delta\left(S_{\infty}\right)^{-1} O(S)$. Then if $\mathbf{p} \notin S, \Phi_{\mathbf{p}}\left(x_{\mathbf{p}} \alpha_{\mathbf{p}}\right)=0$ for all $\alpha_{\mathbf{p}} \in O_{\mathbf{p}}$. Thus if $\alpha \in O(S)$, then (3.1) implies

$$
\operatorname{Tr}_{S}(x \alpha)=\sum_{\mathbf{p} \in S} \Phi_{\mathbf{p}}\left(x_{\mathbf{p}} \alpha_{\mathbf{p}}\right)=0
$$

and thus $x \in \delta(S)^{-1}$ so that $\delta\left(S_{\infty}\right)^{-1} O(S) \subset \delta(S)^{-1}$.

Now suppose $x \notin \delta\left(S_{\infty}\right)^{-1} O(S)$; i.e., $x_{\mathbf{p}} \notin \delta_{\mathbf{p}}^{-1}$ for some $\mathbf{p} \notin S$. Then we can find a $\beta_{\mathbf{p}} \in O_{\mathbf{p}}$ such that $\operatorname{Tr}_{\mathbf{Q}_{\mathbf{p}}}^{K_{\mathbf{p}}}\left(x_{\mathbf{p}} \beta_{\mathbf{p}}\right) \notin \mathbf{Z}_{\mathbf{p}}$. Now by the Chinese Remainder Theorem we can find an $\alpha \in O(S)$ such that $\operatorname{Tr}_{\mathbf{Q}_{q}}^{K_{\mathbf{q}}}\left(x_{\mathbf{q}} \alpha_{\mathbf{q}}\right) \in \mathbf{Z}_{q}$ for all finite $\mathbf{q} \neq \mathbf{p}$ and $\operatorname{Tr}_{\mathbf{Q}_{\mathbf{p}}}^{K_{\mathbf{p}}}\left(x_{\mathbf{p}} \alpha_{\mathbf{p}}\right) \notin \mathbf{Z}_{\mathbf{p}}$. Indeed, this places restrictions on $\alpha$ only at the prime $\mathbf{p}$ (where we require $\alpha_{\mathbf{p}} \equiv \beta_{\mathbf{p}} \bmod \mathbf{p}^{n}$ for $n$ large) and for the finitely many other primes $q$ where $x_{\mathbf{q}} \delta_{\mathbf{q}} \notin O_{\mathbf{q}}$. But then (3.1) implies

$$
\operatorname{Tr}_{S}(x \alpha)=\operatorname{Tr}_{S_{\infty}}(x \alpha)+\sum_{\mathbf{q} \in S_{\text {fin }}} \Phi_{\mathbf{q}}\left(x_{\mathbf{q}} \alpha_{\mathbf{q}}\right)=-\Phi_{\mathbf{p}}\left(x_{\mathbf{p}} \alpha_{\mathbf{p}}\right) \neq 0 .
$$

Thus $x \notin \delta(S)^{-1}$ so that $\delta\left(S_{\infty}\right)^{-1} O(S)=\delta(S)^{-1}$.

We now consider the space we are really interested in for application to Fourier series.

Proposition 4. $\prod_{\mathrm{p} \in S} K_{\mathrm{p}} / O(S)$ is compact and its characters are identified by $x \mapsto \exp \left(2 \pi i \operatorname{Tr}_{S}(\nu x)\right)$ for $\nu \in \delta(S)^{-1}$.

Proof. Let $x=\left(\ldots, x_{\mathbf{p}}, \ldots\right) \in \prod_{\mathbf{p} \in S} K_{\mathbf{p}}$. Then we can find a $t \in O(S)$ such that $x_{\mathbf{p}}-t \in O_{\mathbf{p}}$ for each $\mathbf{p} \in S_{\infty}$ by the Chinese Remainder Theorem. So $x-t \in \prod_{\mathbf{p} \in S_{\infty}} K_{\mathbf{p}} \times \prod_{\mathbf{p} \in S_{\mathrm{fn}}} O_{\mathrm{p}}$. Since the $O_{\mathrm{p}}$ are compact and it is well known that $\prod_{\mathrm{p} \in S_{\infty}} K_{\mathrm{p}} / O\left(S_{\infty}\right)$ is compact, then $\prod_{\mathrm{p} \in S} K_{\mathrm{p}} / O(S)$ is compact as well.

Now from Proposition 2 we look for all $\nu \in \prod_{\mathrm{p} \in S} K_{\mathrm{p}}$ such that $x \mapsto$ $\exp \left(2 \pi i \operatorname{Tr}_{S}(\nu x)\right)$ is trivial on $O(S)$. Suppose $\nu=\left(\ldots, \nu_{\mathrm{p}}, \ldots\right)$ gives such a character. Then choose an $\alpha \in U(S)$ such that $\alpha_{\mathrm{p}} \nu_{\mathrm{p}} \delta_{\mathrm{p}} \in O_{\mathrm{p}}$ for each $\mathbf{p} \in S_{\mathrm{fin}}$. Then $\Phi_{\mathrm{p}}\left(\nu_{\mathrm{p}} \alpha_{\mathrm{p}} x_{\mathrm{p}}\right)=0$ for any $x_{\mathrm{p}} \in O_{\mathrm{p}}$ so in particular

$$
0=\operatorname{Tr}_{S}(\nu \alpha x)=\operatorname{Tr}_{S_{\infty}}(\nu \alpha x)
$$

for any $x \in O\left(S_{\infty}\right)$.

But if $\left\{x_{1},, x_{2}, \ldots, x_{n}\right\}$ is a $\mathbf{Z}$ basis for $O\left(S_{\infty}\right)$, then there is a dual $\mathbf{Z}$ basis $\left\{d_{1}, d_{2}, \ldots, d_{n}\right\}$ of $\delta\left(S_{\infty}\right)^{-1}$ such that

$$
\operatorname{Tr}_{\mathbf{Q}}^{K}\left(d_{i} x_{j}\right)= \begin{cases}1 & \text { if } i=j \\ 0 & \text { if } i \neq j\end{cases}
$$


Since the $d_{i}$ span the $\mathbf{R}$-vector space $\prod_{\mathbf{p} \in S_{\infty}} K_{\mathbf{p}}$, then $\nu_{\infty} \alpha_{\infty}=\sum_{i=1}^{n} m_{i} d_{i}$ for some $m_{i} \in \mathbf{R}$. So

$$
0=\operatorname{Tr}_{S_{\infty}}(\nu \alpha x)=\operatorname{Tr}_{S_{\infty}}\left(\sum m_{i} d_{i} x\right) \equiv \sum m_{i} \operatorname{Tr}_{\mathbf{Q}}^{K}\left(d_{i} x\right) \quad \bmod \mathbf{Z}
$$

for all $x \in O\left(S_{\infty}\right)$. Then taking $x=x_{j}$ shows $m_{j} \equiv 0 \bmod \mathbf{Z}$. Thus $\nu_{\infty} \alpha_{\infty} \in$ $\delta\left(S_{\infty}\right)^{-1}$.

So $\nu_{\infty}=\mu_{\infty}$ for some $\mu \in \delta(S)^{-1}$. Then $\beta=\nu-\mu$ has component $\beta_{\mathrm{p}}=0$ at each infinite $\mathbf{p}$. But still $\operatorname{Tr}_{S}(\beta x)=0$ for all $x \in O(S)$, and since we can choose an $x$ so that $x_{\mathbf{p}}$ has arbitrary order at the $\mathbf{p} \in S_{\mathrm{fin}}$ (by the Chinese Remainder Theorem), this implies $\beta_{\mathbf{p}}=0$ for all $\mathbf{p} \in S_{\mathrm{fin}}$.

Thus $\nu=\mu \in \delta(S)^{-1}$.

With the above characters identified, we can represent a modular form $f$ by its Fourier series

$$
f(z)=\sum_{\nu \in \delta(S)^{-1}} a_{\nu}(y) \exp \left(2 \pi i \operatorname{Tr}_{S}(\nu x)\right)
$$

where the $a_{\nu}(y)$ are of course explicitly representable as integrals. This equality of functions is in the $L^{2}$ sense on $\prod_{p \in S_{0}} K_{p} / O(S)$.

We would, of course, like the Fourier series to converge to $f$ absolutely and uniformly on compact sets. If there were only infinite primes to deal with, this would be simple to show, but unfortunately it is not immediate for more general $S$. We first need

Lemma 5. The individual terms of the Fourier series of

$$
f(z)=\sum_{\nu \in \delta(S)^{-1}} a_{\nu}(y) \exp \left(2 \pi i \operatorname{Tr}_{S}(\nu x)\right)
$$

are locally constant on $H\left(S_{\mathrm{fin}}\right)$.

Proof. Fix some $\mathbf{p} \in S_{\mathrm{fin}}$. Let $\varepsilon=\left(1, \ldots, 1, \varepsilon_{\mathrm{p}}, 1, \ldots, 1\right)$ for some $\varepsilon_{\mathrm{p}} \in U_{\mathbf{p}}$ and let $\alpha=\left(0, \ldots, 0, \alpha_{p}, 0, \ldots, 0\right)$ for some $\alpha_{p} \in O_{p}$. We must show that

$$
a_{\nu}(y \varepsilon) \exp \left(2 \pi i \operatorname{Tr}_{S}(\nu(x+\alpha y))\right)=a_{\nu}(y) \exp \left(2 \pi i \operatorname{Tr}_{S}(\nu x)\right) \text {. }
$$

But

$$
a_{\nu}(y)=\int_{\prod_{\mathrm{p} \in S} K_{\mathrm{p}} / O(S)} f(u+I y) \overline{\exp \left(2 \pi i \operatorname{Tr}_{S}(\nu u)\right)} d u
$$

where $d u$ is the appropriately normalized additive Haar measure on $\prod_{\mathrm{p} \in S} K_{\mathrm{p}}$. Therefore,

$$
\begin{aligned}
& a_{\nu}(y \varepsilon) \exp \left(2 \pi i \operatorname{Tr}_{S}(\nu(x+\alpha y))\right) \\
& \quad=\int_{\prod_{\mathrm{p} \in S} K_{\mathrm{p}} / O(S)} f(u+I \varepsilon y) \overline{\exp \left(2 \pi i \operatorname{Tr}_{S}(\nu u)\right)} d u \exp \left(2 \pi i \operatorname{Tr}_{S}(\nu(x+\alpha y))\right) \\
& \quad=\int f(u+\alpha y+I \varepsilon y) \overline{\exp \left(2 \pi i \operatorname{Tr}_{S}(\nu(u+\alpha y))\right)} d u \exp \left(2 \pi i \operatorname{Tr}_{S}(\nu(x+\alpha y))\right)
\end{aligned}
$$

by translation-invariance of $d u$. But $f(u+\alpha y+I \varepsilon y)=f(u+I y)$ since $f$ is locally constant, so

$$
\begin{aligned}
& =\int f(u+I y) \overline{\exp \left(2 \pi i \operatorname{Tr}_{S}(\nu u)\right)} d u \exp \left(2 \pi i \operatorname{Tr}_{S}(\nu x)\right) \\
& =a_{\nu}(y) \exp \left(2 \pi i \operatorname{Tr}_{S}(\nu x)\right) .
\end{aligned}
$$


Lemma 6. $a_{\nu}(y)$ depends on $y_{\mathbf{p}}$ only up to multiplication by elements of $U_{\mathbf{p}}$ and $a_{\nu}(y)=0$ unless $\delta_{\mathbf{p}} \nu_{\mathbf{p}} y_{\mathbf{p}} \in O_{\mathbf{p}}$ for all $\mathbf{p} \in S_{\text {fin }}$.

Proof. Consider more generally any locally constant function $g: H_{\mathbf{p}} \rightarrow \mathbf{C}$ of the form

$$
g\left(z_{\mathbf{p}}\right)=c\left(y_{\mathbf{p}}\right) \exp \left(2 \pi i \Phi_{\mathbf{p}}\left(\nu_{\mathbf{p}} x_{\mathbf{p}}\right)\right)
$$

where $\nu_{\mathbf{p}} \in K_{\mathbf{p}}$.

By local constancy, $c$ depends only on $\left|y_{\mathbf{p}}\right|_{\mathbf{p}}$ and

$$
g\left(x_{\mathbf{p}}+I_{\mathbf{p}} y_{\mathbf{p}}\right)=g\left(x_{\mathbf{p}}+\alpha y_{\mathbf{p}}+I_{\mathbf{p}} y_{\mathbf{p}}\right)
$$

for any $\alpha \in O_{\mathbf{p}}$. Thus

$$
c\left(y_{\mathbf{p}}\right) \exp \left(2 \pi i \Phi_{\mathbf{p}}\left(\nu_{\mathbf{p}} x_{\mathbf{p}}\right)\right)=c\left(y_{\mathbf{p}}\right) \exp \left(2 \pi i \Phi_{\mathbf{p}}\left(\nu_{\mathbf{p}} x_{\mathbf{p}}\right)\right) \exp \left(2 \pi i \Phi_{\mathbf{p}}\left(\nu_{\mathbf{p}} \alpha y_{\mathbf{p}}\right)\right) .
$$

Therefore if $\Phi_{\mathbf{p}}\left(\nu_{\mathbf{p}} \alpha y_{\mathbf{p}}\right) \neq 0$, then $c\left(y_{\mathbf{p}}\right)=0$; i.e., if $\operatorname{Tr}_{\mathbf{Q}_{\mathbf{p}}}^{K_{\mathbf{p}}}\left(\nu_{\mathbf{p}} \alpha y_{\mathbf{p}}\right) \notin \mathbf{Z}_{\mathbf{p}}$, then $c\left(y_{\mathbf{p}}\right)=0$. Thus $c\left(y_{\mathbf{p}}\right)=0$ unless $\nu_{\mathbf{p}} y_{\mathbf{p}} \in \delta_{\mathbf{p}}^{-1}$. $f$.

We can now return to considering the convergence of the Fourier series for

Lemma 7. The Fourier series $\sum_{\nu \in \delta(S)^{-1}} a_{\nu}(y) \exp \left(2 \pi i \operatorname{Tr}_{S}(\nu x)\right)$ converges to $f$ absolutely and uniformly on compact sets.

Proof. Since $f$ is locally constant in the $z_{\mathbf{p}}$ for finite $\mathbf{p}$, we need only show convergence as stated for any fixed $z_{\text {fin }}$. To this end, let $w=u+I v \in H\left(S_{\text {fin }}\right)$ be fixed and set $g\left(z_{\infty}\right)=f\left(z_{\infty}, w\right)$.

Now $\left.g\right|_{k}\left(\begin{array}{ll}1 & t \\ 0 & 1\end{array}\right)=g$ for any $t \in O(S)$ such that $\left(\begin{array}{ll}1 & t \\ 0 & 1\end{array}\right) \circ M_{w}=M_{w}$; i.e., for any $t \in O(S)$ such that $t_{\mathbf{p}} / v_{\mathbf{p}} \in O_{\mathbf{p}}$ for each $\mathbf{p} \in S_{\mathrm{fin}}$. But this just means $t \in \operatorname{Id}(I v)$. Thus $g$ has a Fourier series and modifying the proof of Proposition 4 to identify the characters on $\prod_{\mathbf{p} \in S_{\infty}} K_{\mathrm{p}} / \operatorname{Id}(I v)$, we see that it is of the form

$$
\sum_{\nu \in \delta\left(S_{\infty}\right)^{-1} \mathbf{I d}(I v)^{-1}} b_{\nu}\left(y_{\infty}\right) \exp \left(2 \pi i \operatorname{Tr}_{S_{\infty}}\left(\nu x_{\infty}\right)\right) .
$$

Now since $g$ is smooth in each of the $z_{\mathbf{p}}$ for infinite $\mathbf{p}$, then in the integrals giving $b_{\nu}$ we can apply the usual differentiation trick to conclude that the Fourier coefficients decay rapidly enough that the Fourier series of $g$ converges absolutely and uniformly to $g$.

We now must relate this to the Fourier series of $f$. We know

$$
\int_{\prod_{\mathrm{p} \in S} K_{\mathrm{p}} / O(S)}\left|f(z)-\sum_{i=1}^{n} a_{\nu_{i}}(y) \exp \left(2 \pi i \operatorname{Tr}_{S}\left(\nu_{i} x\right)\right)\right|^{2} d x \rightarrow 0
$$

as $n \rightarrow \infty$. Therefore

$$
\int_{C}\left|f(z)-\sum_{i=1}^{n} a_{\nu_{i}}(y) \exp \left(2 \pi i \operatorname{Tr}_{S}\left(\nu_{i} x\right)\right)\right|^{2} d x \rightarrow 0
$$

for $C$ any compact set in $\prod_{\mathbf{p} \in S} K_{\mathbf{p}}$. In particular if we take

$$
C=\prod_{\mathbf{p} \in S_{\infty}} K_{\mathbf{p}} / \operatorname{Id}(I v) \times M_{w}
$$


then we see that

$$
\begin{aligned}
\int_{\prod_{\mathrm{p} \in S_{\infty}} K_{\mathrm{p}} / \operatorname{Id}(I v)} \mid f\left(z_{\infty}, w\right)-\sum_{i=1}^{n} a_{\nu_{i}}\left(y_{\infty}, v\right) & \exp \left(2 \pi i \operatorname{Tr}_{S_{\infty}}\left(\nu_{i} x_{\infty}\right)\right) \\
& \times\left.\exp \left(2 \pi i \operatorname{Tr}_{S_{\mathrm{fn}}}\left(\nu_{i} u\right)\right)\right|^{2} d x_{\infty} \rightarrow 0
\end{aligned}
$$

as $n \rightarrow \infty$. Thus

$$
g\left(z_{\infty}\right)=f\left(z_{\infty}, w\right)=\sum_{\nu \in \delta(S)^{-1}} a_{\nu}\left(y_{\infty}, v\right) \exp \left(2 \pi i \operatorname{Tr}_{S_{\infty}}\left(\nu x_{\infty}\right)\right) \exp \left(2 \pi i \operatorname{Tr}_{S_{\text {fin }}}(\nu u)\right)
$$

as $L^{2}$ functions on $\prod_{\mathrm{p} \in S_{\infty}} K_{\mathrm{p}} / \operatorname{Id}(I v)$. Since by Lemma $6 a_{\nu}\left(y_{\infty}, v\right)$ vanishes unless $\delta_{\mathbf{p}} \nu_{\mathbf{p}} v_{\mathbf{p}} \in O_{\mathbf{p}}$ for each $\mathbf{p} \in S_{\mathrm{fin}}$, we have

$$
g\left(z_{\infty}\right)=\sum_{\nu \in \delta\left(S_{\infty}\right)^{-1} \operatorname{Id}(I v)^{-1}} a_{\nu}\left(y_{\infty}, v\right) \exp \left(2 \pi i \operatorname{Tr}_{S_{\infty}}\left(\nu x_{\infty}\right)\right) \exp \left(2 \pi i \operatorname{Tr}_{S_{\text {fin }}}(\nu u)\right)
$$

as $L^{2}$ functions. This, then, must be the Fourier series for $g$ which we already have shown converges as desired. Thus the series for $f$ converges as stated at any fixed $z_{\text {fin }} \in H\left(S_{\text {fin }}\right)$.

From the conditions other than translation invariance that $f$ satisfies, more can be deduced about the Fourier coefficients $a_{\nu}(y)$. We begin with

Lemma 8. $\left|a_{\nu}(y)\right| \leq C\left|\mathbf{N}_{S}(y)\right|^{-k / 2}$ for some $C$ depending only on $f$.

Proof.

$$
a_{\nu}(y)=\int_{\prod_{\mathrm{p} \in S} K_{\mathrm{p}} / O(S)} f(x+I y) \overline{\exp \left(2 \pi i \operatorname{Tr}_{S}(\nu x)\right)} d x .
$$

But $h(z)=N_{S}(y)^{k / 2}|f(z)|$ is invariant under $z \rightarrow A \circ z$ for any $A \in \Gamma_{1}(S)$. Moreover, by the growth condition (2) of the definition of a modular form, $h$ is in fact bounded on a fundamental domain for $\Gamma_{1}(S)$ and hence on all of $H(S)$. Thus $|f(z)| \leq C_{1} \mathbf{N}_{S}(y)^{-k / 2}$ for some $C_{1}$ and therefore the result follows from the above integral.

Again we emphasize that we have restricted our attention to the case $K$ is totally real for simplicity. Since the infinite primes are handled in [R] (or [Te] in the weight 0 case), we merely sketch those cases.

Since $\Delta_{\mathbf{p}} f=\lambda_{\mathbf{p}} f$ for each real $\mathbf{p}$, a calculation and the uniqueness of the Fourier series shows:

$$
\Delta_{\mathrm{p}} a_{\nu}(y) \exp \left(2 \pi i \operatorname{Tr}_{S}(\nu x)\right)=\lambda_{\mathrm{p}} a_{\nu}(y) \exp \left(2 \pi i \operatorname{Tr}_{S}(\nu x)\right) .
$$

But by separation of variables, this means $a_{\nu}(y)$ satisfies a certain second order differential equation in $y_{\mathbf{p}}$. From the fact that $a_{\nu}(y) \rightarrow 0$ as $y_{\mathbf{p}} \rightarrow \infty$ by Lemma 8, the solution can be identified and we are led to conclude that

$$
\begin{gathered}
a_{\nu}(y)=b_{\nu}\left(\left\{y_{\mathbf{q}}\right\}_{\mathbf{q} \in S, \neq \mathbf{p}}\right) y_{\mathbf{p}}^{-k / 2} W_{\mathbf{s g n}(\nu) k / 2, s-1 / 2}\left(4 \pi\left|\nu_{\mathbf{p}}\right| y_{\mathbf{p}}\right) \\
a_{0}(y)=0 .
\end{gathered}
$$


Here $s$ is such that $s(1-s)=\lambda_{\mathrm{p}}-(k / 2)(1-k / 2)$ and $W_{\beta, \mu}(y)$ is the Whittaker function which is the solution of:

$$
\frac{d^{2} W}{d y^{2}}+\left(\frac{-1}{4}+\frac{\beta}{y}+\frac{1 / 4-\mu^{2}}{y^{2}}\right) W=0
$$

and decays exponentially as $y \rightarrow \infty$.

We define the function $W_{\lambda_{\mathbf{p}}}^{\mathbf{p}, k}\left(\nu_{\mathbf{p}} y_{\mathbf{p}}\right)$ to be $\left(\left|\nu_{\mathbf{p}}\right| y_{\mathbf{p}}\right)^{-k / 2} W_{\operatorname{sgn}(\nu) k / 2, s-1 / 2}\left(4 \pi\left|\nu_{\mathbf{p}}\right| y_{\mathbf{p}}\right)$.

The finite primes we treat in more detail. Fix any $\mathbf{p} \in S_{\mathrm{fin}}$.

Lemma 9. The individual terms of the Fourier series of $f$ are eigenfunctions of $T_{\mathrm{p}}$ with eigenvalues $\lambda_{\mathrm{p}}$.

Proof. For any function $g\left(z_{\mathbf{p}}\right)=a\left(y_{\mathbf{p}}\right) \exp \left(2 \pi i \Phi_{\mathbf{p}}\left(\nu_{\mathbf{p}} x_{\mathbf{p}}\right)\right): H_{\mathbf{p}} \rightarrow \mathbf{C}$, where $\nu_{\mathbf{p}} \in$ $K_{\mathbf{p}}$,

$$
\begin{aligned}
T_{\mathbf{p}}\left(z_{\mathbf{p}}\right)= & |\pi| \sum_{i=1}^{p^{f}} a\left(\pi y_{\mathbf{p}}\right) \exp \left(2 \pi i \Phi_{\mathbf{p}}\left(\nu_{\mathbf{p}}\left(x_{\mathbf{p}}+\alpha_{i} y_{\mathbf{p}}\right)\right)\right) \\
& +|\pi|^{1-k} a\left(y_{\mathbf{p}} \pi^{-1}\right) \exp \left(2 \pi i \Phi_{\mathbf{p}}\left(\nu_{\mathbf{p}} x_{\mathbf{p}}\right)\right) \\
= & b\left(y_{\mathbf{p}}\right) \exp \left(2 \pi i \Phi_{\mathbf{p}}\left(\nu_{\mathbf{p}} x_{\mathbf{p}}\right)\right)
\end{aligned}
$$

for some $b\left(y_{\mathbf{p}}\right)$. Applying this to

$$
f(z)=\sum_{\nu \in \delta(S)^{-1}} a_{\nu}(y) \exp \left(2 \pi i \operatorname{Tr}_{S}(\nu x)\right)
$$

yields

$$
T_{\mathbf{p}} f(z)=\sum b_{\nu}(y) \exp \left(2 \pi i \operatorname{Tr}_{S}(\nu x)\right)
$$

where $b_{\nu}(y)$ is given by

$$
T_{\mathbf{p}}\left(a_{\nu}(y) \exp \left(2 \pi i \operatorname{Tr}_{S}(\nu x)\right)\right)=b_{\nu}(y) \exp \left(2 \pi i \operatorname{Tr}_{S}(\nu x)\right)
$$

But since $T_{\mathbf{p}} f=\lambda_{\mathbf{p}} f$, the uniqueness of the Fourier series implies that

$$
T_{\mathbf{p}}\left(a_{\nu}(y) \exp \left(2 \pi i \operatorname{Tr}_{S}(\nu x)\right)\right)=\lambda_{\mathrm{p}} a_{\nu}(y) \exp \left(2 \pi i \operatorname{Tr}_{S}(\nu x)\right) .
$$

Consider any locally constant function $g\left(z_{\mathbf{p}}\right)=c\left(y_{\mathbf{p}}\right) \exp \left(2 \pi i \Phi_{\mathbf{p}}\left(\nu_{\mathbf{p}} x_{\mathbf{p}}\right)\right)$ : $H_{\mathrm{p}} \rightarrow \mathrm{C}$, where $\nu_{\mathrm{p}} \in K_{\mathrm{p}}$ such that $T_{\mathrm{p}} g=\lambda_{\mathrm{p}} g$.

By the proof of Lemma 6 if $\delta_{p} \nu_{p} y_{p} \notin O_{p}$ then $c\left(y_{p}\right)=0$. Letting $d\left(y_{p}\right)=$ $c\left(\delta_{\mathbf{p}}^{-1} \nu_{\mathbf{p}}^{-1} y_{\mathbf{p}}\right)$, we have $d\left(y_{\mathbf{p}}\right)=0$ if $y_{\mathbf{p}} \notin O_{\mathbf{p}}$ and

$$
g\left(z_{\mathbf{p}}\right)=d\left(\delta_{\mathbf{p}} \nu_{\mathbf{p}} y_{\mathbf{p}}\right) \exp \left(2 \pi i \Phi_{\mathbf{p}}\left(\nu_{\mathbf{p}} x_{\mathbf{p}}\right)\right)
$$

Since $T_{\mathbf{p}} g=\lambda_{\mathrm{p}} g$ and

$$
\begin{aligned}
T_{\mathbf{p}} g\left(z_{\mathbf{p}}\right)= & |\pi|_{\mathbf{p}} \sum_{i=1}^{p^{f}} d\left(\delta_{\mathbf{p}} \nu_{\mathbf{p}} \pi y_{\mathbf{p}}\right) \exp \left(2 \pi i \Phi_{\mathbf{p}}\left(\nu_{\mathbf{p}}\left(x_{\mathbf{p}}+\alpha_{i} y_{\mathbf{p}}\right)\right)\right) \\
& +|\pi|_{\mathbf{p}}^{1-k} d\left(\delta_{\mathbf{p}} \nu_{\mathbf{p}} \pi^{-1} y_{\mathbf{p}}\right) \exp \left(2 \pi i \Phi_{\mathbf{p}}\left(\nu_{\mathbf{p}} x_{\mathbf{p}}\right)\right)
\end{aligned}
$$

we see that when $\delta_{\mathrm{p}} \nu_{\mathrm{p}} y_{\mathrm{p}} \in O_{\mathrm{p}}$ and therefore $\Phi_{\mathbf{p}}\left(\nu_{\mathrm{p}} \alpha_{i} y_{\mathrm{p}}\right)=0$, then

$$
\begin{aligned}
& \lambda_{\mathbf{p}} d\left(\delta_{\mathbf{p}} \nu_{\mathbf{p}} y_{\mathbf{p}}\right) \exp \left(2 \pi i \Phi_{\mathbf{p}}\left(\nu_{\mathbf{p}} x_{\mathbf{p}}\right)\right) \\
& \quad=\left(d\left(\delta_{\mathbf{p}} \nu_{\mathbf{p}} \pi y_{\mathbf{p}}\right)+|\pi|_{\mathbf{p}}^{1-k} d\left(\delta_{\mathbf{p}} \nu_{\mathbf{p}} \pi^{-1} y_{\mathbf{p}}\right)\right) \exp \left(2 \pi i \Phi_{\mathbf{p}}\left(\nu_{\mathbf{p}} x_{\mathbf{p}}\right)\right) .
\end{aligned}
$$


Therefore, if $y_{\mathbf{p}} \in O_{\mathbf{p}}$, then

$$
\lambda_{\mathbf{p}} d\left(y_{\mathbf{p}}\right)=d\left(\pi y_{\mathbf{p}}\right)+|\pi|_{\mathbf{p}}^{1-k} d\left(\pi^{-1} y_{\mathbf{p}}\right) .
$$

Definition. The weight $k$ p-adic Whittaker function of eigenvalue $\lambda_{\mathbf{p}}$ is $W_{\lambda_{\mathbf{p}}}^{\mathbf{p}, k}$ : $K_{\mathbf{p}}^{\times} \rightarrow \mathrm{C}$ defined by

$$
W_{\lambda_{\mathbf{p}}}^{\mathbf{p}, k}\left(y_{\mathbf{p}}\right)= \begin{cases}0 & \text { if } \operatorname{ord}_{\mathbf{p}} y_{\mathbf{p}}<0 \\ 1 & \text { if } \operatorname{ord}_{\mathbf{p}} y_{\mathbf{p}}=0\end{cases}
$$

and

$$
\lambda_{\mathbf{p}} W_{\lambda_{\mathbf{p}}}^{\mathbf{p}, k}\left(y_{\mathbf{p}}\right)=W_{\lambda_{\mathbf{p}}}^{\mathbf{p}, k}\left(\pi y_{\mathbf{p}}\right)+|\pi|_{\mathbf{p}}^{1-k} W_{\lambda_{\mathbf{p}}}^{\mathbf{p}, k}\left(\pi^{-1} y_{\mathbf{p}}\right) \quad \text { for } \operatorname{ord}_{\mathbf{p}} y_{\mathbf{p}} \geq 0
$$

With this definition, we have that $d\left(y_{\mathbf{p}}\right)=d(1) W_{\lambda_{\mathbf{p}}}^{\mathbf{p}, k}\left(y_{\mathbf{p}}\right)$.

Applying the above discussion to the Fourier series of $f$ yields

Lemma 10.

$$
f(z)=\sum_{\nu \in \delta(S)^{-1}} b_{\nu}\left(\left\{y_{\mathbf{q}}\right\}_{\mathbf{q} \in S, \neq \mathbf{p}}\right) W_{\lambda_{\mathbf{p}}}^{\mathbf{p}, k}\left(\delta_{\mathbf{p}} \nu_{\mathbf{p}} y_{\mathbf{p}}\right) \exp \left(2 \pi i \operatorname{Tr}_{S}(\nu x)\right) .
$$

Combining this result with the earlier one for infinite $\mathbf{p}$ gives

Proposition 11. For $y_{\mathbf{p}}>0$ for each infinite $\mathbf{p}, f$ has Fourier series

$$
f(z)=\sum_{\nu \in \delta(S)^{-1}} a_{\nu} \mathbf{W}_{\Lambda}^{S, k}(\delta \nu y) \exp \left(2 \pi i \operatorname{Tr}_{S}(\nu x)\right)
$$

where $\mathbf{W}_{\Lambda}^{S, k}(y)=\prod_{\mathbf{p} \in S} W_{\lambda_{\mathbf{p}}}^{\mathbf{p}, k}\left(y_{\mathbf{p}}\right)$ and $\delta=\left(\ldots, \delta_{\mathbf{p}}, \ldots\right)$ where we set $\delta_{\mathbf{p}}=1$ if $\mathbf{p} \in S_{\infty}$ and otherwise $\delta_{\mathbf{p}}$ is the local different. This series converges absolutely and uniformly on compact sets.

If we consider two sets of primes $S_{1} \subset S_{2}$ containing $S_{\infty}$, then $\delta\left(S_{1}\right)^{-1} \subset$ $\delta\left(S_{2}\right)^{-1}$ and thus there are "more" terms in the Fourier series and hence "more" Fourier coefficients for an $S_{2}$ form than for an $S_{1}$ form.

But $\left.f\right|_{k}\left(\begin{array}{cc}\varepsilon & 0 \\ 0 & \varepsilon^{-1}\end{array}\right)=f$ for any $\varepsilon \in U^{+}(S)$ means

$$
\begin{aligned}
& \sum_{\nu \in \delta(S)^{-1}} a_{\nu} \mathbf{W}_{\Lambda}^{S, k}\left(\delta \nu \varepsilon^{2} y\right) \exp \left(2 \pi i \operatorname{Tr}_{S}\left(\nu \varepsilon^{2} x\right)\right) \\
& =\sum_{\nu \in b(S)^{-1}} a_{\nu} \mathbf{W}_{\Lambda}^{S, k}(\delta \nu y) \exp \left(2 \pi i \operatorname{Tr}_{S}(\nu x)\right)
\end{aligned}
$$

Equating Fourier coefficients shows $a_{\nu}=a_{\nu \varepsilon^{2}}$. Thus the presence of additional coefficients is deceptive since many are the same and therefore contain no new information.

Lemma 8 can also be used to say something about the growth of the p-adic Whittaker function that occurs in the Fourier coefficients of a cusp form $f$.

Lemma 12. If $f$ is a cusp form with $T_{\mathbf{p}} f=\lambda_{\mathbf{p}} f$, then $\left|W_{\lambda_{\mathbf{p}}}^{\mathbf{p}, k}\left(y_{\mathbf{p}}\right)\right| \leq C\left|y_{\mathbf{p}}\right|_{\mathbf{p}}^{-k / 2}$ for some $C$ depending only on $f$.

From Lemma 8

$$
\left|a_{\nu} \mathbf{W}_{\Lambda}^{S, k}(\delta \nu y)\right| \leq C_{1} \mathbf{N}_{S}(y)^{-k / 2}
$$


For each $\mathbf{q} \in S, \mathbf{q} \neq \mathbf{p}$, choose $y_{\mathbf{q}}$ so that $W_{\lambda_{\mathbf{p}}}^{\mathbf{p}, k}\left(\delta_{\mathbf{q}} \nu_{\mathbf{q}} y_{\mathbf{q}}\right) \neq 0$. Then

$$
\left|W_{\lambda_{\mathbf{p}}}^{\mathbf{p}, k}\left(\delta_{\mathbf{p}} \nu_{\mathbf{p}} y_{\mathbf{p}}\right)\right| \leq C_{2}\left|y_{\mathbf{p}}\right|_{\mathbf{p}}^{-k / 2}
$$

so

$$
\left|W_{\lambda_{\mathbf{p}}}^{\mathbf{p}, k}\left(y_{\mathbf{p}}\right)\right| \leq C_{3}\left|y_{\mathbf{p}}\right|_{\mathbf{p}}^{-k / 2}
$$

\section{THE DiRICHLET SERIES ASSOCIATED WITH A MODULAR FORM}

Let $f \in \mathbf{S}_{k}\left(\Gamma_{1}(S), \Lambda\right)$ for some totally real number field $K$. To simplify the analysis at the infinite primes, let us assume that $f$ is actually holomorphic in each $z_{\mathbf{p}}$ for the infinite $\mathbf{p}$ (i.e., $\lambda_{\mathbf{p}}=0$ for all $\mathbf{p} \in S_{\infty}$ ). If this is not the case, the necessary modifications are well known from any work on Maass forms.

From §III, for each $\mathbf{p} \in S_{\infty}$ we therefore have that

$$
W_{\lambda_{\mathbf{p}}}^{\mathbf{p}, k}\left(\nu_{\mathbf{p}} y_{\mathbf{p}}\right) \exp \left(2 \pi i \Phi_{\mathbf{p}}\left(\nu_{\mathbf{p}} x_{\mathbf{p}}\right)\right)= \begin{cases}\exp \left(2 \pi i \nu_{\mathbf{p}} z_{\mathbf{p}}\right) & \text { if } \nu_{\mathbf{p}}>0, \\ 0 & \text { otherwise }\end{cases}
$$

so

$$
f(z)=\sum_{\substack{\nu \in \delta(S)^{-1} \\ \nu \gg 0}} a_{\nu} \mathbf{W}_{\Lambda}^{S, k}(\delta \nu y) \exp \left(2 \pi i \operatorname{Tr}_{S}(\nu x)\right)
$$

where $\nu \gg 0$ means $\nu$ is totally positive and $W_{\lambda_{\mathbf{p}}}^{\mathbf{p}, k}\left(\nu_{\mathbf{p}} y_{\mathbf{p}}\right)=\exp \left(-2 \pi \nu_{\mathbf{p}} y_{\mathbf{p}}\right)$ for $\mathbf{p} \in S_{\infty}$.

Recalling that $a_{\nu}=a_{\nu \varepsilon^{2}}$ for any $\varepsilon \in U^{+}(S)$, it is natural to consider the formal Dirichlet series

$$
L(f, s)=\sum_{\nu \in \delta(S)^{-1} / U^{+}(S)^{2}} \frac{a_{\nu}}{\mathbf{N}_{S}(\nu)^{s}} .
$$

In order to discuss convergence of this series we must first understand the growth of the $a_{\nu}$.

Lemma 1. $\left|a_{\nu}\right| \leq C \mathbf{N}_{S}(\nu)^{k / 2}$ for some constant $C$ depending only $f$.

Proof. From Lemma 8 of $\S$ III

$$
\left|a_{\nu} \mathbf{W}_{\Lambda}^{S, k}(\nu y)\right| \leq C_{1} \mathbf{N}_{S}(y)^{-k / 2} .
$$

Now choosing any vector $a$ such that $\mathbf{W}_{\Lambda}^{S, k}(a) \neq 0$ and then letting $y=a / \nu$ we get $\left|a_{\nu}\right| \leq C \mathbf{N}_{S}(\nu)^{k / 2}$ for some constant $C$.

Lemma 2. The series defining $L(f, s)$ converges absolutely and uniformly on compact sets for $\operatorname{Re}(s)>k / 2+1$.

Proof. In light of Lemma 1, we must show that

$$
\sum_{\substack{\nu \in \delta(S)^{-1} / U^{+}(S)^{2} \\ \nu \gg 0}} \mathbf{N}_{S}(\nu)^{-\sigma}
$$

converges for $\sigma>1$. But this is majorized by

$$
\sum_{\nu \in \delta(S)^{-1} / U(S)}\left|\mathbf{N}_{S}(\nu)\right|^{-\sigma} \leq \sum_{\mathbf{a}} \mathbf{N}_{\mathbf{Q}}^{K}(\mathbf{a})^{-\sigma}
$$


where the sum is over fractional ideals a of $O\left(S_{\infty}\right)$ such that $\mathbf{a} \delta\left(S_{\infty}\right)$ is integral and a has no prime factors appearing in $S$. This in turn is majorized by

$$
\mathbf{N}_{\mathbf{Q}}^{K}\left(\delta\left(S_{\infty}\right)\right)^{\sigma} \sum_{\mathbf{a} \subset O\left(S_{\infty}\right)} \mathbf{N}_{\mathbf{Q}}^{K}(\mathbf{a})^{-\sigma}
$$

where we sum over all integral ideals, and this last is just the Dedekind zeta function of $K$ which is well known to converge for $\sigma>1$.

In preparation for discussing the analytic continuation and functional equation of $L(f, s)$, we must first introduce the appropriate Gamma functions for each prime $\mathbf{p} \in S$.

First let us fix the notation for some groups and measures we will be using. Let

$$
K_{\mathbf{p}}^{*}= \begin{cases}\mathbf{R}^{+}, & \text {the positive reals, } \\ K_{\mathbf{p}}^{\times} & \text {if } \mathbf{p} \text { is real, } \\ \text { if } \mathbf{p} \text { is finite }\end{cases}
$$

We use $d y_{\mathbf{p}}^{\times}$to denote the multiplicative Haar measure on $K_{\mathbf{p}}^{*}$ normalized so that $d y_{\mathbf{p}}^{\times}=d y_{\mathbf{p}} / y_{\mathbf{p}}$ for $\mathbf{p}$ real and $d y_{\mathbf{p}}^{\times}$gives $U_{\mathbf{p}}$ mass 1 for $\mathbf{p}$ finite.

Definition. For any prime $\mathbf{p} \in S$, let $\Gamma_{\mathbf{p}}(s)=\int_{K_{\mathbf{p}}^{*}} W_{\lambda_{\mathbf{p}}}^{\mathbf{p}, k}\left(\delta_{\mathbf{p}} y_{\mathbf{p}}\right)\left|y_{\mathbf{p}}\right|_{\mathbf{p}}^{s} d y_{\mathbf{p}}^{\times}$.

A change of variables shows that for $\mathbf{p}$ real

$$
\Gamma_{\mathbf{p}}(s)=(2 \pi)^{-s} \int_{0}^{\infty} \exp (-y) y^{s} \frac{d y}{y}=(2 \pi)^{-s} \Gamma(s)
$$

where $\Gamma(s)$ is the classical $\Gamma$ function. Thus $\Gamma_{\mathbf{p}}(s)$ has an analytic continuation to the whole complex plane and is well understood.

For $\mathbf{p}$ finite, a change of variables gives

$$
\begin{aligned}
\Gamma_{\mathbf{p}}(s) & =\left|\delta_{\mathbf{p}}\right|_{\mathbf{p}}^{-s} \int_{K_{\mathbf{p}}^{\times}} W_{\lambda_{\mathbf{p}}}^{\mathbf{p}, k}\left(y_{\mathbf{p}}\right)\left|y_{\mathbf{p}}\right|^{s} d y_{\mathbf{p}}^{\times} \\
& =\left|\delta_{\mathbf{p}}\right|_{\mathbf{p}}^{-s}\left(1+W_{\lambda_{\mathbf{p}}}^{\mathbf{p}, k}(\pi)|\pi|^{s}+W_{\lambda_{\mathbf{p}}}^{\mathbf{p}, k}\left(\pi^{2}\right)\left|\pi^{2}\right|^{s}+\cdots\right) .
\end{aligned}
$$

Now from Lemma 12 of $\S I I I,\left|W_{\lambda_{\mathrm{p}}}^{\mathbf{p}, k}\left(\pi^{n}\right)\right| \leq C\left|\pi^{n}\right|^{-k / 2}$, so this series is majorized by $\sum_{n=1}^{\infty}\left|\pi^{n}\right|_{\mathbf{p}}^{s-k / 2}$ which converges for $\operatorname{Re}(s)>k / 2$. But since

$$
\lambda_{\mathbf{p}} W_{\lambda_{\mathbf{p}}}^{\mathbf{p}, k}\left(y_{\mathbf{p}}\right)=W_{\lambda_{\mathbf{p}}}^{\mathbf{p}, k}\left(\pi y_{\mathbf{p}}\right)+|\pi|^{1-k} W_{\lambda_{\mathbf{p}}}^{\mathbf{p}, k}\left(\pi^{-1} y_{\mathbf{p}}\right)
$$

then for $s$ sufficiently large

$$
\left(1-\lambda_{\mathbf{p}}|\pi|^{s}+|\pi|^{1-k+2 s}\right)\left(1+W_{\lambda_{\mathbf{p}}}^{\mathbf{p}, k}(\pi)|\pi|^{s}+W_{\lambda_{\mathbf{p}}}^{\mathbf{p}, k}\left(\pi^{2}\right)\left|\pi^{2}\right|^{s}+\cdots\right)=1 .
$$

Thus an analytic continuation of $\Gamma_{\mathbf{p}}(s)$ is given by

$$
\Gamma_{\mathbf{p}}(s)=\left|\delta_{\mathbf{p}}\right|^{-s}\left(1-\lambda_{\mathbf{p}}|\pi|^{s}+|\pi|^{1-k+2 s}\right)^{-1}
$$

Note that the dependence of $\Gamma_{\mathbf{p}}(s)$ on $\lambda_{\mathbf{p}}$ has been dropped from the notation.

Theorem 3. If $f \in \mathbf{S}_{k}\left(\Gamma_{1}(S), \Lambda\right)$ is holomorphic, define

$$
R(f, s)=\prod_{\mathbf{p} \in S} \Gamma_{\mathbf{p}}(s) L(f, s) .
$$


Then $R(f, s)$ continues to an entire function on the whole complex plane which satisfies the functional equation

$$
R(f, s)=i^{n k} R(f, k-s)
$$

where $n=[K: \mathbf{Q}]$.

Proof. Consider

$$
R_{0}(f, s)=\int_{\prod_{\mathbf{p} \in S} K_{\mathbf{p}}^{*} / U^{+}(S)^{2}} f(I y) \mathbf{N}_{S}(y)^{s} d y_{S}^{\times}
$$

where $d y_{S}^{\times}=\prod_{\mathbf{p} \in S} d y_{\mathbf{p}}^{\times}$.

First, since the integrand is invariant under $y \rightarrow \varepsilon^{2} y$ for $\varepsilon \in U^{+}(S)$, this is well defined provided that it converges.

At least formally we have

$$
\begin{aligned}
R_{0}(f, s) & =\int_{\prod_{\mathbf{p} \in S} K_{\mathbf{p}}^{*} / U^{+}(S)^{2}}\left(\sum_{\substack{\nu \in \delta(S)^{-1} \\
\nu \gg 0}} a_{\nu} \mathbf{W}_{\Lambda}^{S, k}(\delta \nu y) \mathbf{N}_{S}(y)^{s}\right) d y_{S}^{\times} \\
& =\int_{\prod_{\mathbf{p} \in S} K_{\mathbf{p}}^{*}}\left(\sum_{\substack{\nu \in \delta(S)^{-1} / U^{+}(S)^{2} \\
\nu \gg 0}} a_{\nu} \mathbf{W}_{\Lambda}^{S, k}(\delta \nu y) \mathbf{N}_{S}(y)^{s}\right) d y_{S}^{\times} \\
& =\sum_{\nu \in \delta(S)^{-1} / U^{+}(S)^{2}} a_{\nu \gg 0} \int_{\prod_{\mathbf{p} \in S} K_{\mathbf{p}}^{*}} \mathbf{W}_{\Lambda}^{S, k}(\delta \nu y) \mathbf{N}_{S}(y)^{s} d y_{S}^{\times} \\
& =\sum_{\nu \in \delta(S)^{-1} / U^{+}(S)^{2}} a_{\nu \gg 0} \mathbf{N}_{S}(\nu)^{-s} \int_{\prod_{\mathbf{p} \in S} K_{\mathbf{p}}^{*}} \mathbf{W}_{\Lambda}^{S, k}(\delta y) \mathbf{N}_{S}(y)^{s} d y_{S}^{\times} \\
& =\sum_{\nu \in \delta(S)^{-1} / U^{+}(S)^{2}} a_{\nu \gg 0} \mathbf{N}_{S}(\nu)^{-s} \prod_{\mathbf{p} \in S} \int_{K_{\mathbf{p}}^{*}} W_{\Lambda_{\mathbf{p}}}^{\mathbf{p}, k}\left(\delta_{\mathbf{p}} y_{\mathbf{p}}\right)\left|y_{\mathbf{p}}\right|_{\mathbf{p}}^{s} d y_{\mathbf{p}}^{\times} \\
& =\prod_{\mathbf{p} \in S} \Gamma_{\mathbf{p}}(s) L(f, s) .
\end{aligned}
$$

Now for $\operatorname{Re}(s)>k / 2+1$ from Lemma 2 and the discussion of the $\Gamma$ functions, both the integrals and the sum in (4.2) converge absolutely. This justifies the interchange of the sum and integral at (4.1) and shows both the absolute and uniform convergence of the original integral and that $R_{0}(f, s)=R(f, s)$ when $\operatorname{Re}(s)>k / 2+1$.

We now make a change of variables in the integral defining $R_{0}(f, s)$. Fix some infinite prime $\mathbf{q}$ and let $S_{0}=S-\{\mathbf{q}\}$. Then consider $f(I y)$ as a function of $u$ and $y_{\mathbf{p}}$ for all $\mathbf{p} \in S_{0}$, where $u=\mathbf{N}_{S}(y) \in \mathbf{R}^{+}$so that $y_{\mathbf{q}}=u / \mathbf{N}_{S_{0}}(y)$. 
Then

$$
\begin{aligned}
R(f, s)= & \int_{u \in \mathbf{R}^{+}}\left(\int_{\prod_{\mathbf{p} \in S_{0}} K_{\mathbf{p}}^{*} / U^{+}(S)^{2}} f(I y) d y_{S_{0}}^{\times}\right) u^{s} \frac{d u}{u} \\
= & \int_{u=0}^{1}\left(\int_{\prod_{\mathbf{p} \in S_{0}} K_{\mathbf{p}}^{*} / U^{+}(S)^{2}} f(I y) d y_{S_{0}}^{\times}\right) u^{s} \frac{d u}{u} \\
& +\int_{u=1}^{\infty}\left(\int_{\prod_{\mathrm{p} \in S_{0}} K_{\mathrm{p}}^{*} / U^{+}(S)^{2}} f(I y) d y_{S_{0}}^{\times}\right) u^{s} \frac{d u}{u} \\
= & I_{1}+I_{2}
\end{aligned}
$$

where both of these integrals converge absolutely and uniformly on compact sets for $\operatorname{Re}(s)>k / 2+1$. Actually $I_{2}$ converges absolutely and uniformly on compact sets for all $s \in \mathbf{C}$ (by comparison with a large real $s$ ), and thus defines an entire function.

Replacing $u \rightarrow 1 / u$ and $y \rightarrow 1 / y$ in $I_{1}$ gives

$$
I_{1}=\int_{u=1}^{\infty}\left(\int_{\prod_{\mathrm{p} \in S_{0}} K_{\mathrm{p}}^{*} / U^{+}(S)^{2}} f\left(I y^{-1}\right) d y_{S_{0}}^{\times}\right) u^{-s} \frac{d u}{u}
$$

But $f=\left.f\right|_{k}\left(\begin{array}{cc}0 & -1 \\ 1 & 0\end{array}\right)$ implies that

$$
f(I y)=f\left(I y^{-1}\right) \mathbf{N}_{S}(I y)^{-k}
$$

Since $\mathbf{N}_{S}(I y)=i^{n} \mathbf{N}_{S}(y)$,

$$
I_{1}=i^{n k} \int_{u=1}^{\infty}\left(\int_{\prod_{\mathrm{p} \in S_{0}} K_{\mathrm{p}}^{*} / U^{+}(S)^{2}} f(I y) d y_{S_{0}}^{\times}\right) u^{k-s} \frac{d u}{u} .
$$

This defines an entire function exactly as $I_{2}$. Thus $R(f, s)$ extends to an entire function.

Moreover, it is clear from this integral representation of $R(f, s)$ that

$$
R(f, s)=i^{n k} R(f, k-s) \text {. }
$$

Written out in full

$$
\begin{aligned}
R(f, s)= & \prod_{\mathbf{p} \in S_{\infty}}(2 \pi)^{-s} \Gamma(s) \prod_{\mathbf{p} \in S_{\text {fin }}}\left|\delta_{\mathbf{p}}\right|_{\mathbf{p}}^{-s}\left(1-\lambda_{\mathbf{p}}|\pi|^{s}+|\pi|^{1}-k-2 s\right)^{-1} \\
& \times \sum_{\substack{\nu \in \delta(S)^{-1} / U^{+}(S)^{2} \\
\nu \gg 0}} \frac{a_{\nu}}{\mathbf{N}_{S}(\nu)^{s}} .
\end{aligned}
$$

Now if $S$ contains all primes appearing in the global different $\delta\left(S_{\infty}\right)$, then $\delta(S)=O(S)$. Moreover, if $D_{\mathbf{Q}}^{K}$ denotes the discriminant, then

$$
\left|D_{\mathbf{Q}}^{K}\right|=\mathbf{N}_{\mathbf{Q}}^{K}\left(\delta\left(S_{\infty}\right)\right)=\left(\prod_{\mathbf{p} \text { finite }}\left|\delta_{\mathbf{p}}\right|_{\mathbf{p}}\right)^{-1}=\left(\prod_{\mathbf{p} \in S_{\text {fin }}}\left|\delta_{\mathbf{p}}\right|_{\mathbf{p}}\right)^{-1}
$$


so that

$$
\begin{aligned}
R(f, s)= & \left((2 \pi)^{-n}\left|D_{\mathbf{Q}}^{K}\right|\right)^{s} \Gamma(s)^{n} \prod_{\mathbf{p} \in S_{\text {fin }}}\left(1+W_{\Lambda_{\mathbf{p}}}^{\mathbf{p}, k}(\pi)|\pi|^{s}+W_{\Lambda_{\mathbf{p}}}^{\mathbf{p}, k}\left(\pi^{2}\right)\left|\pi^{2}\right|^{s}+\cdots\right) \\
& \times \sum_{\substack{\nu \in \delta(S)^{-1} / U^{+}(S)^{2} \\
\nu \gg 0}} \frac{a_{\nu}}{\mathbf{N}_{S}(\nu)^{s}} \\
= & \left((2 \pi)^{-n}\left|D_{\mathbf{Q}}^{K}\right|\right)^{s} \Gamma(s)^{n} \sum_{\mathbf{a} \subset O\left(S_{\infty}\right)} \frac{b(\mathbf{a})}{\mathbf{N}_{\mathbf{Q}}^{K}(\mathbf{a})^{s}}
\end{aligned}
$$

for appropriate numbers $b(\mathbf{a})$. In fact, if we merely dropped the condition on $f$ that $T_{\mathrm{p}} f=\lambda_{\mathrm{p}} f$ we could have reached this point (but of course we would not be able to relate the $b(\mathbf{a})$ to the Fourier coefficients of $f$ as nicely).

On the other hand, if we wish to pull other Euler factors out of the series $L(f, s)$, then we can either add more primes to $S$, or develop a theory of Hecke operators for primes not in $S$. Provided that $S$ has been chosen so that $h(S)=1$, there is no difficulty in following this last course almost exactly as in [Sh], though some care must be taken since we want to associate a Hecke operator to an ideal and not to any of its generators. This will be done in $\S \mathrm{VI}$.

\section{RESTRICTING AND EXTENDING $S$}

Suppose that for a totally real field $K$ we consider two finite sets of prime $S_{0}, S_{1}$, such that $S_{1} \supset S_{0} \supset S_{\infty}$. Then if $\tilde{f} \in \mathbf{S}_{k}\left(\Gamma_{U}\left(S_{1}\right), \chi_{1}, \Lambda_{1}\right)$ so that $\tilde{f}: H\left(S_{1}\right) \rightarrow \mathbf{C}$, then it is easily seen that if we let $f(z)=\tilde{f}(z, w): H\left(S_{0}\right) \rightarrow \mathbf{C}$ where $w=\left(\ldots, I_{\mathbf{p}}, \ldots\right)$ as $\mathbf{p}$ runs through $S_{1}-S_{0}$, then

$$
f \in \mathbf{S}_{k}\left(\Gamma_{U}\left(S_{0}\right), \chi_{0}, \Lambda_{0}\right)
$$

where $\chi_{0}$ is just the restriction of $\chi_{1}$ to $U\left(S_{0}\right)$ and $\Lambda_{0}$ is the subset of $\Lambda_{1}$ corresponding to the primes in $S_{0}$. To see this one need only note that if $A \in \Gamma_{U}\left(S_{0}\right)$ then $A \circ M_{w}=M_{w}$.

The question then arises whether this process can be reversed; that is, can an $S_{0}$-form be extended to an $S_{1}$-form? To do this we must first decompose the space $\mathbf{S}_{k}\left(\Gamma_{U}\left(S_{0}\right), \chi_{0}, \Lambda_{0}\right)$.

First we note that $\Gamma_{U}\left(S_{0}\right)$ can be enlarged by trivial actions. Since for $\varepsilon \in$ $U^{+}\left(S_{1}\right)$ we see that $\left.\right|_{k}\left(\begin{array}{ll}\varepsilon & 0 \\ 0 & \varepsilon\end{array}\right)$ acts trivially on any element of $\mathbf{S}_{k}\left(\Gamma_{U}\left(S_{0}\right), \chi_{0}, \Lambda_{0}\right)$, then $\mathbf{S}_{k}\left(\Gamma_{U}\left(S_{0}\right), \chi_{0}, \Lambda_{0}\right)=\mathbf{S}_{k}\left(\Gamma_{0}, \phi_{0}, \Lambda_{0}\right)$, where $\Gamma_{0}$ is the group generated by $\Gamma_{U}\left(S_{0}\right)$ and matrices of this form, and $\phi_{0}$ extends $\chi_{0}$ and is 1 on elements of $U^{+}\left(S_{1}\right)^{2}$.

Let $\Gamma_{w}=\left\{A \in \Gamma_{U}\left(S_{1}\right) \mid A \circ M_{w}=M_{w}\right\}$ with $w$ as above. Then by Corollary 4 of $\S \mathbf{I}$, if $A \in \Gamma_{w}$ then for each $\mathbf{p} \in S_{1}-S_{0}$,

$$
A=\left(\begin{array}{cc}
\pi^{n} & 0 \\
0 & \pi^{n}
\end{array}\right) A_{1}
$$

where $A_{1} \in G L_{2}\left(O_{\mathbf{p}}\right)$. In fact, if we set $V_{w}=\operatorname{det}\left(\Gamma_{w}\right) \subset U\left(S_{1}\right)$ and $V_{0}=$ $\operatorname{det}\left(\Gamma_{0}\right)$, then the sequence

$$
0 \rightarrow \Gamma_{0} \rightarrow \Gamma_{w} \rightarrow V_{w} / V_{0} \rightarrow 0
$$

is exact. Since $V_{w} / V_{0}$ is finite and abelian, then

$$
\mathbf{S}_{k}\left(\Gamma_{U}\left(S_{0}\right), \chi_{0}, \Lambda_{0}\right)=\bigoplus_{\phi} \mathbf{S}_{k}\left(\Gamma_{w}, \phi, \Lambda_{0}\right)
$$


where $\phi$ runs through characters of $V_{w}$ extending $\phi_{0}$ and $f \in \mathbf{S}_{k}\left(\Gamma_{w}, \phi, \Lambda_{0}\right)$ means that $f \in \mathbf{S}_{k}\left(\Gamma_{U}\left(S_{0}\right), \chi_{0}, \Lambda_{0}\right)$ and $\left.f\right|_{k} A=\phi(\operatorname{det}(A)) f$ for any $A \in \Gamma_{w}$.

Now for $f \in \mathbf{S}_{k}\left(\Gamma_{w}, \phi, \Lambda_{0}\right)$, define $\tilde{f}: H\left(S_{0}\right) \times M_{w} \subset H\left(S_{1}\right) \rightarrow \mathbf{C}$ by $\tilde{f}(z, \tau)=f(z)$ for any $\tau \in M_{w}$. Then if $\chi_{1}$ is any character on $U\left(S_{1}\right)$ extending $\phi$ then we can use the condition $\left.\tilde{f}\right|_{k} A=\chi_{1}(\operatorname{det}(A)) \tilde{f}$ to extend $f$ to a function $\tilde{f}$ on $H\left(S_{0}\right) \times\left(\Gamma_{U}\left(S_{1}\right) \circ M_{w}\right) \subset H\left(S_{1}\right)$. We note that such $\chi_{1}$ are easy to construct since $U\left(S_{1}\right) / V_{w}$ is finite.

Unfortunately, as the examination of the fundamental domain in §II, Proposition 2 showed, $H\left(S_{0}\right) \times\left(\Gamma_{U}\left(S_{1}\right) \circ M_{w}\right)$ is not necessarily all of $H\left(S_{1}\right)$. Therefore we set $\tilde{f}(z)=0$ for all $z \in H\left(S_{1}\right)$ where we have not yet defined it.

Note that for $\mathbf{p} \in S_{0}$, we have $T_{\mathbf{p}} \tilde{f}=\left(\widetilde{T_{\mathbf{p}} f}\right)$ since $T_{\mathbf{p}}$ acts only in the $z_{\mathbf{p}}$ variable and commutes with the slash operator. A look back at the definition of a modular form shows that $\tilde{f}$ satisfies all the conditions except possibly (3b) for those primes $\mathbf{p} \in S_{1}-S_{0}$. That is, we do not know whether $T_{\mathbf{p}} f=\lambda_{\mathbf{p}} f$ for some $\lambda_{\mathbf{p}} \in \mathbf{C}$ for these primes. We will have to return to this problem in the next section.

In particular we note that the above process allows classical Hilbert modular forms (i.e., $S_{\infty}$-forms) to be brought into the theory of $S$-forms for larger $S$. Then, once we diagonalize the action of the $T_{\mathbf{p}}$ for $\mathbf{p} \in S-S_{\infty}$ this will show the existence of $S$-forms.

Finally, it is worth pointing out that it is necessary that our modular forms be locally constant on the $H_{\mathbf{p}}$ for finite $\mathbf{p}$ so that this extension process works. Since $\Gamma_{U}\left(S_{0}\right) \circ I_{\mathbf{p}}$ is dense in $M_{I_{\mathrm{p}}}$ for any $\mathbf{p} \notin S_{0}$, then if $f$ is modular on $\Gamma_{U}\left(S_{0}\right)$, its extension to $H_{i}\left(S_{0}\right) \times H_{\mathbf{p}}$ must be constant as $z_{\mathbf{p}}$ ranges through $M_{I_{\mathrm{p}}}$ and hence through any $M_{\xi_{\mathrm{p}}}$.

\section{HECKE OPERATORS THROUGH DOUBLE COSETS}

Hecke operators are usually defined directly in terms of double cosets, so that while it is clear there is a connection to the $T_{\mathbf{p}}$ as defined here, it has not been made fully explicit yet.

Let $S_{\infty} \subset S_{0} \subset S_{1}$ be finite sets of primes of a totally real field $K$. Suppose further that $h\left(S_{0}\right)=1$ and chose any $\mathbf{p} \in S_{1}-S_{0}$. Then $T_{\mathbf{p}}$ can be defined as in $\S I$ for $S_{1}$-forms and defined via double cosets for $S_{0}$-forms.

One easily proves

Lemma 1. Let $S_{0} \supset S_{\infty}$ be such that $h\left(S_{0}\right)=1$. Let $\mathbf{p}=(\pi)$ as an $O\left(S_{0}\right)$ ideal for some $\mathbf{p} \notin S_{0}$. Then

$$
\Gamma_{1}\left(S_{0}\right)\left(\begin{array}{cc}
\pi & 0 \\
0 & 1
\end{array}\right) \Gamma_{1}\left(S_{0}\right)=\bigcup_{i} \Gamma_{1}\left(S_{0}\right)\left(\begin{array}{cc}
1 & \alpha_{i} \\
0 & \pi
\end{array}\right) \cup \Gamma_{1}\left(S_{0}\right)\left(\begin{array}{cc}
\pi & 0 \\
0 & 1
\end{array}\right)
$$

where the $\alpha_{i}$ run through a complete set of representatives of $O\left(S_{0}\right) / \mathbf{p}$, is a disjoint double coset decomposition.

Now we can consider the double coset Hecke operator given (except for normalization) by

$$
\left.f\right|_{k, S_{0}} \Gamma_{1}\left(S_{0}\right)\left(\begin{array}{cc}
\pi & 0 \\
0 & 1
\end{array}\right) \Gamma_{1}\left(S_{0}\right)=\left.\sum_{j} f\right|_{k, S_{0}} A_{j}
$$


where the $A_{j}$ run through a complete set of right coset representatives of the double coset. This is clearly independent of the choice of the $A_{j}$.

Proposition 2. Under the conditions above, if $\tilde{f} \in \mathbf{S}_{k}\left(\Gamma_{U}\left(S_{1}\right), \chi_{1}, \Lambda_{1}\right)$ and $f(z)=\tilde{f}(z, w) \in \mathbf{S}_{k}\left(\Gamma_{U}\left(S_{0}\right), \chi_{0}, \Lambda_{0}\right)$ is its restriction as in $\S \mathrm{V}$, then

$$
\left(T_{\mathbf{p}} \tilde{f}\right)(z, w)=\left.\bar{\chi}_{1}(\pi)|\pi|_{\mathbf{p}}^{1-k / 2} f\right|_{k, S_{0}} \Gamma_{1}\left(S_{0}\right)\left(\begin{array}{cc}
\pi & 0 \\
0 & 1
\end{array}\right) \Gamma_{1}\left(S_{0}\right)(z) .
$$

Proof. By Lemma 1

$$
\begin{aligned}
&\left.\bar{\chi}_{1}(\pi)|\pi|_{\mathbf{p}}^{1-k / 2} f\right|_{k, S_{0}} \Gamma_{1}\left(S_{0}\right)\left(\begin{array}{cc}
\pi & 0 \\
0 & 1
\end{array}\right) \Gamma_{1}\left(S_{0}\right)(z) \bar{\chi}_{1}(\pi)|\pi|_{\mathbf{p}}^{1-k / 2}\left[\left.\sum_{i} f\right|_{k, S_{0}}\left(\begin{array}{cc}
1 & \alpha_{i} \\
0 & \pi
\end{array}\right)(z)+\left.f\right|_{k, S_{0}}\left(\begin{array}{cc}
\pi & 0 \\
0 & 1
\end{array}\right)(z)\right] \\
&= \bar{\chi}_{1}(\pi)|\pi|_{\mathbf{p}}^{1-k / 2}\left[\sum_{i} f\left(\left(\begin{array}{cc}
1 & \alpha_{i} \\
0 & \pi
\end{array}\right) \circ z\right) \mathbf{N}_{S_{0}}(\pi)^{-k / 2}\right. \\
&\left.+f\left(\left(\begin{array}{cc}
\pi & 0 \\
0 & 1
\end{array}\right) \circ z\right) \mathbf{N}_{S_{0}}(\pi)^{k / 2}\right] \\
&= \bar{\chi}_{1}(\pi)\left[|\pi|_{\mathbf{p}} \sum_{i} \tilde{f}\left(\left(\begin{array}{cc}
1 & \alpha_{0} \\
0 & \pi
\end{array}\right) \circ z, w\right) \mathbf{N}_{S_{1}}(\pi)^{-k / 2}\right. \\
&\left.+|\pi|_{\mathbf{p}}^{1-k} \tilde{f}\left(\left(\begin{array}{cc}
\pi & 0 \\
0 & 1
\end{array}\right) \circ z, w\right) \mathbf{N}_{S_{1}}(\pi)^{k / 2}\right]
\end{aligned}
$$

Now since $\left.\tilde{f}\right|_{k, S_{1}} A=\chi_{1}(\operatorname{det}(A)) \tilde{f}$ for $A \in \Gamma_{U}\left(S_{1}\right)$,

$$
\begin{aligned}
& =\bar{\chi}_{\mathbf{1}}(\pi) \chi_{1}(\pi)\left[|\pi|_{\mathbf{p}} \sum_{i} \tilde{f}\left(z,\left(\begin{array}{cc}
1 & \alpha_{i} \\
0 & \pi
\end{array}\right)^{-1} \circ w\right) \mathbf{N}_{S}(\pi)^{-k / 2} \mathbf{N}_{S}\left(\pi^{-1}\right)^{-k / 2}\right. \\
& \left.+|\pi|_{\mathbf{p}}^{1-k} \tilde{f}\left(z,\left(\begin{array}{cc}
\pi & 0 \\
0 & 1
\end{array}\right)^{-1} \circ w\right) \mathbf{N}_{S}(\pi)^{k / 2} \mathbf{N}_{S}\left(\pi^{-1}\right)^{k / 2}\right] \\
& =\left(T_{\mathbf{p}} \tilde{f}\right)(z, w)
\end{aligned}
$$

since $w=\left(\ldots, I_{\mathbf{p}}, \ldots\right)$.

This makes clear that it is unimportant from the standpoint of Hecke operators whether additional primes are killed off by being included in $S$ after $h(S)=1$ already. Thus in the case $h(S)=1$, a full theory relating modular forms to Dirichlet series with Euler products can be developed. One also sees immediately from this that all of the $T_{\mathbf{p}}$ commute, regardless of their definition in terms of double cosets or integral operators since they can be viewed as integral operators which obviously commute.

If $S_{\infty} \subset S_{0} \subset S_{1}$ are finite, $\chi_{1}$ is a character of $U\left(S_{1}\right)$, and $\Lambda_{0}$ is an eigenvalue system for $S_{0}$, let $\mathbf{S}_{k}\left(\Gamma_{U}\left(S_{1}\right), \chi_{1}, \Lambda_{0}\right)$ be the vector space of functions satisfying all the requirements to be in $\mathbf{S}_{k}\left(\Gamma_{U}\left(S_{1}\right), \chi_{1}, \Lambda_{1}\right)$ except possibly that there is no $\lambda_{\mathbf{p}}$ such that $T_{\mathbf{p}} f=\lambda_{\mathbf{p}} f$ for $\mathbf{p} \notin S_{0}$. 
We wish to show that

$$
\mathbf{S}_{k}\left(\Gamma_{U}\left(S_{1}\right), \chi_{1}, \Lambda_{0}\right)=\bigoplus_{\Lambda_{1}} \mathbf{S}_{k}\left(\Gamma_{U}\left(S_{1}\right), \chi_{1}, \Lambda_{1}\right)
$$

where $\Lambda_{1}$ runs through eigenvalue systems for $S_{1}$ extending $\Lambda_{0}$. In order to do this we will need two facts: (1) $\mathbf{S}_{k}\left(\Gamma_{U}\left(S_{1}\right), \chi_{1}, \Lambda_{0}\right)$ is finite dimensional; and (2) $\mathbf{S}_{k}\left(\Gamma_{U}\left(S_{1}\right), \chi_{1}, \Lambda_{0}\right)$ has a scalar product with respect to which $T_{\mathrm{p}}$ is Hermitian. If these two conditions are met then since the operators $\left\{T_{\mathbf{p}}\right\}$ commute, they can be simultaneously diagonalized, and our space of forms decomposes as desired.

From investigating the fundamental domain of $\Gamma_{U}\left(S_{1}\right)$ in Proposition 2 of $\S$ II, we know that $f \in \mathbf{S}_{k}\left(\Gamma_{U}\left(S_{1}\right), \chi_{1}, \Lambda_{0}\right)$ is fully determined by a finite number of classical Hilbert modular forms, modular with respect to the groups $\Gamma_{\mathbf{a}_{i}}$. As does Herrmann, we cite the work of Maass to see that the spaces of forms on such $\Gamma_{\mathbf{a}_{i}}$ (in fact on subgroups of these) is finite dimensional (at least in the holomorphic case, though the proof should generalize). Thus we leave (1) as settled.

(2) is more involved, but again reduces to standard results. We first must define a scalar product.

Definition. The Petersson scalar product on $\mathbf{S}_{k}\left(\Gamma_{U}\left(S_{1}\right), \chi, \Lambda_{0}\right)$ is defined by

$$
\langle f, g\rangle_{\Gamma_{U}\left(S_{1}\right)}=\int_{F_{1}} f(z) \overline{g(z)} \mathbf{N}_{S_{1}}(y)^{k} d z
$$

where $F_{1}$ is a fundamental domain for $\Gamma_{U}\left(S_{1}\right)$ and $d z=\prod_{\mathbf{p} \in S} d z_{\mathbf{p}}$ where

$$
d z_{\mathbf{p}}= \begin{cases}y_{\mathbf{p}}^{-2} d x_{\mathbf{p}} d y_{\mathbf{p}} & \text { if } \mathbf{p} \text { is real } \\ d \mu\left(z_{\mathbf{p}}\right) \text { of } \S \mathbf{I} & \text { if } \mathbf{p} \text { is finite }\end{cases}
$$

Since $d z$ is $G L_{2}(K)$ invariant and the integrand is $\Gamma_{U}\left(S_{1}\right)$ invariant, one sees that $\langle f, g\rangle_{\Gamma_{U}\left(S_{1}\right)}$ does not depend on the choice of the fundamental domain. By the growth conditions on cusp forms, this integral actually converges, and one checks that it satisfies all the requirements for a scalar product.

Lemma 3. Suppose $\tilde{f}, \tilde{g} \in \mathbf{S}_{k}\left(\Gamma_{U}\left(S_{1}\right), \chi_{1}, \Lambda_{0}\right)$ and let $\mathbf{p} \in S_{1}$ be finite. Suppose in addition that $S_{1}$ is large enough that if $S_{0}=S_{1}-\{\mathbf{p}\}$, then $h\left(S_{0}\right)=1$. Then

$$
\left\langle T_{\mathbf{p}} \tilde{f}, \tilde{g}\right\rangle_{\Gamma_{U}\left(S_{1}\right)}=\left\langle\tilde{f}, T_{\mathbf{p}} \tilde{g}\right\rangle_{\Gamma_{U}\left(S_{1}\right)} .
$$

Proof. Let $\hat{z} \in H\left(S_{0}\right)$ be $z \in H\left(S_{1}\right)$ with the $z_{\mathrm{p}}$ component deleted. Let $f(\hat{z})=\tilde{f}\left(\hat{z}, I_{\mathbf{p}}\right), g(\hat{z})=\tilde{g}\left(\hat{z}, I_{\mathbf{p}}\right)$. Then

$$
\left\langle T_{\mathbf{p}} \tilde{f}, \tilde{g}\right\rangle_{\Gamma_{U}\left(S_{1}\right)}=\int_{F_{1}}\left(T_{\mathbf{p}} \tilde{f}\right)(z) \overline{\tilde{g}(z)} \mathbf{N}_{S_{1}}(y)^{k} d z .
$$

But since $h\left(S_{0}\right)=1, F_{1}$ can be taken to have the form $F \times M_{I_{\mathrm{p}}}$ where $F \subset$ $H\left(S_{0}\right)$ is a fundamental domain for

$$
\Gamma=\left\{A \in \Gamma_{U}\left(S_{1}\right) \mid A \circ M_{I_{\mathrm{p}}}=M_{I_{\mathrm{p}}}\right\} \supset \Gamma_{U}\left(S_{0}\right) .
$$


Note that $F$ is also a fundamental domain for $\Gamma_{U}\left(S_{0}\right)$. So

$$
\begin{aligned}
\left\langle T_{\mathbf{p}} \tilde{f}, \tilde{g}\right\rangle_{\Gamma_{U}\left(S_{1}\right)} & =\int_{F_{1}}\left(T_{\mathbf{p}} \tilde{f}\right)\left(\hat{z}, z_{\mathbf{p}}\right) \overline{\tilde{g}\left(\hat{z}, z_{\mathbf{p}}\right)} \mathbf{N}_{S_{1}}\left(y, y_{\mathbf{p}}\right)^{k} d z \\
& =\int_{F}\left(T_{\mathbf{p}} \tilde{f}\right)\left(\hat{z}, I_{\mathbf{p}}\right) \overline{\tilde{g}\left(\hat{z}, I_{\mathbf{p}}\right)} \mathbf{N}_{S_{0}}(\hat{y})^{k} d z \\
& =\int_{F}\left(T_{\mathbf{p}} f\right)(\hat{z}) \overline{g(\hat{z})} \mathbf{N}_{S_{0}(\hat{y})^{k} d z} \\
& =\left\langle T_{\mathbf{p}} f, g\right\rangle_{\Gamma_{U}\left(S_{0}\right) .}
\end{aligned}
$$

But since $h\left(S_{0}\right)=1$, by Proposition 2 we have

$$
=\overline{\chi_{1}(\pi)}|\pi|_{\mathbf{p}}^{1-k / 2}\left\langle\left.\sum_{i} f\right|_{k} A_{i}, g\right\rangle_{\Gamma_{U}\left(S_{0}\right)}
$$

where the $A_{i}$ run through some choice of double coset representatives.

The proof can now proceed exactly as the usual proof for Hecke operators defined in terms of double cosets (cf. [Sh]). We omit the rather involved but standard argument.

It is of course disappointing to rely on the classical double coset definition of Hecke operators in this proof, particularly when one considers Proposition 12 of $\S$ I. Regardless, we have

Corollary 4. Let $S_{1}$ be large enough such that for any finite $\mathbf{p} \in S_{1}$, if we let $S_{0}=S_{1}-\{\mathbf{p}\}$, then $h\left(S_{0}\right)=1$. Then

$$
\mathbf{S}_{k}\left(\Gamma_{U}\left(S_{1}\right), \chi_{1}, \Lambda_{0}\right)=\bigoplus_{\Lambda_{1}} \mathbf{S}_{k}\left(\Gamma_{U}\left(S_{1}\right), \chi_{1}, \Lambda_{1}\right) .
$$

Corollary 5. For any $S_{1}$

$$
\mathbf{S}_{k}\left(\Gamma_{U}\left(S_{1}\right), \chi_{1}, \Lambda_{0}\right)=\bigoplus_{\Lambda_{1}} \mathbf{S}_{k}\left(\Gamma_{U}\left(S_{1}\right), \chi_{1}, \Lambda_{1}\right) .
$$

Proof. By $\S \mathrm{V}$ any $f, g \in \mathbf{S}_{k}\left(\Gamma_{U}\left(S_{1}\right), \chi_{1}, \Lambda_{0}\right)$ are the linear combinations of restrictions to $H\left(S_{1}\right)$ of elements of $\mathbf{S}_{k}\left(\Gamma_{U}\left(S_{2}\right), \phi_{i}, \Lambda_{0}\right)$ where $S_{2}$ is a large enough set of primes that $O\left(S_{2}-\{\mathbf{p}\}\right)$ has class number one for all $\mathbf{p} \in S_{2}$ and $\phi_{i}$ extend $\chi_{1}$. Since these spaces have a basis of eigenforms of $T_{\mathrm{p}}$ and since $T_{\mathbf{p}}$ behaves well with respect to restricting forms back to $\mathbf{S}_{k}\left(\Gamma_{U}\left(S_{1}\right), \chi_{1}, \Lambda_{0}\right)$, that space has a basis of eigenforms as well.

\section{HERRMANN'S THEORY}

In this section we will briefly show how one of the vector modular forms defined in $[\mathrm{H}]$ is related to an $S$-modular form. However, our goal is not to restate all of the definitions and results of that paper. Therefore, the reader unfamiliar with the paper will find this discussion a bit sketchy.

We begin by defining a vector modular form. Rather than introduce ideal numbers as Herrmann does, we give an equivalent formulation which will be less notationally cumbersome. 
Let $K$ be a totally real number field and fix some choice $\left\{\mathbf{a}_{i}\right\}_{i=1}^{h}, h=h\left(S_{\infty}\right)$, of ideal class representatives of $O\left(S_{\infty}\right)$. Let

$$
\Gamma_{1, \mathbf{a}_{i}}=\left\{\left(\begin{array}{ll}
a & b \\
c & d
\end{array}\right) \in G L_{2}(K) \mid a, d \in O\left(S_{\infty}\right), b \in \mathbf{a}_{i}, c \in \mathbf{a}_{i}^{-1}, a d-b c=1\right\}
$$

(as in $\S I I)$,

$$
\begin{aligned}
& \Gamma_{U, \mathbf{a}_{i}}=\left\{\left(\begin{array}{ll}
a & b \\
c & d
\end{array}\right) \in G L_{2}(K) \mid a, d \in O\left(S_{\infty}\right), \quad b \in \mathbf{a}_{i}, \quad c \in \mathbf{a}_{i}^{-1},\right. \\
& \left.\quad a d-b c \in U\left(S_{\infty}\right)\right\}, \\
& \Gamma_{2, \mathbf{a}_{i}}=\left\{\left(\begin{array}{ll}
a & b \\
c & d
\end{array}\right) \in G L_{2}(K) \mid a, d \in \mathbf{b}, b \in \mathbf{a}_{i} \mathbf{b}, \quad c \in \mathbf{a}_{i}^{-1} \mathbf{b},\right. \\
& \left.(a d-b c)=\mathbf{b}^{2} \text { for some ideal } \mathbf{b}\right\} .
\end{aligned}
$$

Definition. The $\mathbf{a}_{i}$-component of a weight $k$ vector modular form is a function $f: H\left(S_{\infty}\right) \rightarrow$ C satisfying

(1) $f$ is holomorphic on $H\left(S_{\infty}\right)$.

(2) $\left.f\right|_{k} A=f$ for all $A \in \Gamma_{1, \mathbf{a}_{i}}$.

(3) $f$ is "bounded at the cusps".

A vector modular form $F$ is a vector $\left\{f_{i}\right\}_{i=1}^{h}$ where each $f_{i}$ is an $\mathbf{a}_{i}$ component. Such an $F$ is said to be of type $\{K,-k\}$.

For any character $\chi$ of $U\left(S_{\infty}\right), F$ is said to be of type $\{K,-k, \chi\}$ if $F$ is of type $\{K,-k\}$ and in addition $\left.f_{i}\right|_{k} A=\chi(\operatorname{det}(A)) f_{i}$ for any $A \in \Gamma_{U, \mathbf{a}_{i}}$. As usual, the space of forms of type $\{K,-k\}$ decomposes into a direct sum over $\chi$ of the spaces of forms of type $\{K,-k, \chi\}$.

Unfortunately, these are still not the forms for which Hecke theory can be developed. In a rather complicated step that involves extending $\chi$ to all the ideal numbers and introducing a character $\psi$ of the ideal class group, Herrmann decomposes the space of forms of type $\{K,-k, \chi\}$ into a direct sum of spaces of forms of type $\{K,-k, \chi, \psi\}$. For our purposes it is enough to know that if $F$ is of type $\{K,-k, \chi, \psi\}$, then

$$
\left.f_{i}\right|_{k} A=\chi(\operatorname{det}(A)) f_{i} \quad \text { for all } A \in \Gamma_{2, \mathbf{a}_{i}}
$$

and there is some consistency among the components of $F$, in that if the class of $\mathbf{a}_{i}$ differs from the class of $\mathbf{a}_{j}$ by a square, then $f_{i}$ determines $f_{j}$. Thus, though Herrmann does not state it explicitly, we do not really need all $h$ components of $F$, but only as many as there are elements in the ideal class group modulo squares. (This fits with de Bruijn's thesis result mentioned in the introduction.)

But now using the discussion of fundamental domains in $\S \mathrm{II}$, it is easy to construct an $S$-form from a form $F$ of type $\{K,-k, \chi, \psi\}$. Let $S$ be the set of all primes dividing any of the $\mathbf{a}_{i}$ as well as all infinite primes (so $h(S)=1$ ). Renumber the $\mathbf{a}_{i}$ if necessary so that $\left\{\mathbf{a}_{i}\right\}_{i=1}^{m}$ is a complete set of representatives of the ideal class group modulo squares. We note that $\chi$ is now defined on all of $K$ and hence in particular on $U(S)$. 
Now define $f_{F}: H(S) \rightarrow \mathrm{C}$ by defining it first on the fundamental domain for $\Gamma_{U}(S)$ of Proposition 2 of $\S$ II by

$$
f_{F}\left(z_{\infty}, \tau\right)=f_{i}\left(z_{\infty}\right) \text { for any } \tau \in M_{w\left(\mathbf{a}_{i}\right)}, i=1,2,3, \ldots, m,
$$

and then extending $f_{F}$ to all of $H(S)$ by the modularity condition

$$
\left.f_{F}\right|_{k} A=\chi(\operatorname{det}(A)) f_{F} \quad \text { for } A \in \Gamma(S) \text {. }
$$

Since $\Gamma_{\mathbf{a}_{i}} \subset \Gamma_{2, \mathbf{a}_{i}}$, then by (7.1) $f_{F}$ is well defined and one easily checks that it has the properties required of a modular form, except possibly that it is not an eigenform of the $T_{p}$ for $p \in S_{\text {fin }}$. However, that issue has already been discussed.

\section{REFERENCES}

[dB] N. G. de Bruijn, Over modulaire vormen van meer veranderlijken, Thesis, Free University of Amsterdam, 1943

[G1] K.-B. Gundlach, Poincaresche und Eisensteinsche Reihen zur Hilbertschen Modulgruppe, Math. Z. 64 (1956), 339-352.

[G2] _ Dirichletsche Reihen zur Hilbertschen Modulgruppe, Math. Ann. 135 (1958), 294-314.

[H] O. Herrmann, Uber Hilbertsche Modulfunctionen und die Dirichletschen Reihen mit Eulerschen Producktentwicklung, Math. Ann. 127 (1954), 357-400.

[La] S. Lang, Algebraic number theory, Addison-Wesley, New York, 1970.

[R] W. Roelke, Das Eigenwertproblem der automorphen Formen in der hyperbolischen Ebene. I, II, Math. Ann. 167 (1966), 292-337; 168 (1967), 261-324.

[Sa] P. Sarnak, The arithmetic and geometry of some hyperbolic three manifolds, Acta Math. 151 (1984), 253-295.

[Se] J. P. Serre, Trees, Springer-Verlag, Berlin and New York, 1980.

[Sc] A. Schwartz, Modular forms of weight $\frac{1}{2}$ defined on products of p-adic upper half-planes, Trans. Amer. Math. Soc. 335 (1993), 757-773.

[Si] C. L. Siegel, Lectures on advanced analytic number theory, Tata Institute Lectures, Bombay, 1961.

[Sh] G. Shimura, Arithmetic theory of automorphic functions, Princeton Univ. Press, Princeton, NJ, 1971.

[St] H. M. Stark, Modular forms and related objects, Number Theory (Proc. 1985 Montreal Conf.), Amer. Math. Soc., Providence, RI, 1987, pp. 421-455.

[Sr] R. Styer, Hecke theory over arbitrary number fields, J. Number Theory 33 (1989), 107-131.

[T] J. Tate, Fourier analysis in number fields and Hecke's zeta-functions, Algebraic Number Theory, Thompson, Washington, DC, 1967, pp. 305-347.

[Te] A. Terras, Harmonic analysis on symmetric spaces and applications. I, Springer-Verlag, New York, 1985.

[W] A. Weil, Dirichlet series and automorphic forms, Lecture Notes in Math., vol. 189, SpringerVerlag, Berlin and New York, 1971.

Department of Mathematics, Bates College, Lewiston, Maine 04240

E-mail address: jrhodescabacus . bates.edu 